\title{
The differential involvement of the prelimbic and infralimbic cortices in response conflict affects behavioral flexibility in rats trained in a new automated strategy-switching task
}

\author{
Catherine Oualian ${ }^{1,2}$ and Pascale Gisquet-Verrier ${ }^{1,2,3}$ \\ ${ }^{1}$ CNRS, Centre de Neurosciences Paris-Sud, UMR 8195, Orsay F-91405, France; ${ }^{2}$ University Paris-Sud, UMR 8195, Orsay F-91405, \\ France
}

To assess the role of the prelimbic (PL) and infralimbic (IL) cortices in mediating strategy switching, rats were trained in a new automated task in a Y-maze allowing a careful analysis of rats' behavior. In this situation, rats can only use two egocentric (Right, Left) and two visual (Light, Dark) strategies. In the first experiment, rats with PL, IL, or PL/IL lesions were compared with sham-operated rats when trained to reach a criterion of 10 consecutive correct responses with a light strategy before being trained with a response strategy (rule shifting), and finally with the reversed response strategy (reversal). In the second experiment, sham-operated and PL-lesioned rats had their first two strategy switches in the reverse order, which was followed by a second rule shifting and reversal. The results indicate that lesions did not affect initial acquisition, but impaired the first rule shifting and reversal. Thorough analyses of rats' performance indicate that lesioned rats were still able to demonstrate some behavioral flexibility but have difficulties in solving response conflicts, which in turn may affect behavioral flexibility. Both areas were differentially involved in the resolution of response conflict, with the IL involved in the choice of strategy previously known to be nonvalid, and the PL in the selection and maintenance of that strategy.

Behavioral flexibility corresponding to the ability to adapt one's behavior to changes in the environment is essential for every individual survival. Flexible behaviors are compromised in a myriad of neuropsychiatric disorders, including schizophrenia, depression, and attention deficit disorder (Goldberg and Weinberger 1988; Morice 1990; Deweer et al. 1994; Beats et al. 1996; Fossati et al. 1999; Merriam et al. 1999; Cahill et al. 2001; Lawrence et al. 2004). In humans, strategy-shifting ability is usually investigated by using the Wisconsin Card Sorting Test (WCST) (Milner 1963) in which subjects have to consecutively sort cards on the basis of different visual dimensions (form, number, or color). Most studies investigating behavioral flexibility in rodents have used either an attentional set-shifting or a strategy-switching task.

The attentional set-shifting task was first developed in humans and primates (Roberts et al. 1988) and subsequently adapted for rats by Birrell and Brown (2000). In this task, rats are trained with stimuli that differ along several perceptual dimensions (bowls differing by three different dimensions: odor, texture, and digging medium) with discrimination possibly based upon one dimension and then another (extra-dimensional shift), or upon two variants of the same dimension (intradimensional shift). This very elegant task, based on simple choices, is easy to acquire, and all the different stages are administered in a single test session. The task is always used with the same procedure in which rats repeatedly learned to ignore one dimension before being required to use it. In addition, as changes always occur simultaneously with the introduction of new exemplars, the situation does not remain the same during the test. Both of these

${ }^{3}$ Corresponding author.

E-mail pascale.gisquet@u-psud.fr; fax 33-(0)1-69-15-77-26.

Article is online at http://www.learnmem.org/cgi/doi/10.1101/lm.1858010. elements of procedure prevent conflicts between responses and preclude possibilities to perseverate on previous stimuli.

In strategy-shifting tasks, rats are usually trained in a crossmaze to use one discrimination to find food (for instance, a visual discrimination) and then to shift to another discrimination based on a different dimension (such as an egocentric strategy). This task is quite a complex task for several reasons: First, the stimuli remain constant across the different phases; second, rats may use different strategies to solve the task and not only those selected by the experimenter such as spatial, cue, or alternation of response strategies. Finally, the difficulty of the task also relies on the complexity of the reward contingencies. For example, when rats are trained with the Light strategy, this strategy is fully reinforced, while the opposite one, the Dark strategy, is unreinforced. Under these conditions, each response strategy (turning right or left) is associated with 50\% of reinforced trials, as the light is inevitably associated with a particular location during half of the trials, and strategy switching is linked with a reorganization of the reward contingencies. An advantage of this task is to allow determining the type of errors made by rats where it is possible to dissociate perseverative, regressive, and never reinforced errors. However, the task requires rearrangement of stimuli from trial to trial, changes in the situation during the probe test, and complex analyses to determine different types of errors (Ragozzino et al. 1999a; Stefani et al. 2003). In addition, this task does not provide any clue about the way rats learned the different discriminations.

Prefrontal cortex damage impairs rule or strategy shifting in humans, monkeys, and rodents (Owen et al. 1991; Dias et al. 1996; Ragozzino et al. 1999a; Gisquet-Verrier et al. 2000). There is evidence that separate areas of the prefrontal cortex differentially contribute to different forms of behavioral flexibility. For 
instance, reversal learning, which corresponds to reverse stimulus reward associations, has been shown to be sensitive to orbitofrontal cortex lesions and not to medial prefrontal cortext (mPFC) lesions, while lesions or inactivations of mPFC in rodents have been reported to disrupt the acquisition of rule-shifting but have no effect on reversal (Ragozzino et al. 1999a; Birrell and Brown 2000; Floresco et al. 2008). It seems, however, that factors such as task difficulty, or level of response conflict, may question these assertions (Bussey et al. 1997; Floresco et al. 2008).

Previous studies indicated that two mPFC subregions, prelimbic (PL) and infralimbic (IL) cortices, are more particularly involved in strategy switching. With the attentional set-shifting task, a role for the PL/IL area in extra- but not intradimensional shifts has been shown, however, without characterizing the processes responsible for the disruption (Birrell and Brown 2000; Tait et al. 2009). With the cross-maze task, the involvement of $\mathrm{PL} / \mathrm{IL}$ in strategy switching has only been studied with transient inactivations and not with lesions. Several studies report that PL/IL inactivation left initial discrimination and reversal learning intact but disrupted the acquisition of rule-shifting (Ragozzino et al. 1999a, 2003; Birrell and Brown 2000; Rich and Shapiro 2007; Floresco et al. 2008; Tait et al. 2009). They further indicated that PL/IL inactivation induces perseveration of the previously learned strategy, suggesting that rats had difficulties in inhibiting the previously learned strategy (Ragozzino et al. 1999a; Block et al. 2007). The PL/IL region has not systematically been associated with perseveration on what has previously been learned (Delatour and Gisquet-Verrier 1996, 1999, 2000), and the possibility remains that rats with lesions perseverate because, due to the task, maintaining the initial strategy still leads to food for half of the trials.

PL and IL cortices are two adjacent cortical areas with different cytoarchitecture (Van Eden and Uylings 1985) and despite large interconnections, they partly differed in their connections with other brain areas (Hoover and Vertes 2007). Over the past few years, several studies have suggested a functional dichotomy between these two areas (Chudasama and Robbins 2003; Killcross and Coutureau 2003; Murphy et al. 2005; Quirk and Beer 2006; Vidal-Gonzalez et al. 2006; Kalivas and O'Brien 2008; Peters et al. 2008). To date, however, PL and IL cortices have been associated with behavioral flexibility without distinguishing between the two areas (Granon and Poucet 1995; Delatour and Gisquet-Verrier 1996; Ragozzino et al. 1999b), although there is some recent evidence showing different neuronal activity in these two areas during rule switching (Rich and Shapiro 2009).

The aim of the present experiment is thus to study the effect of combined and separated PL and IL lesions during strategy switching, using a task allowing us to clearly determine the nature of the disrupted processes. Here we have developed an automated strategy-switching task in which rats can autonomously perform successive discriminations. The task derived from a fully automated brightness discrimination task in a Y-maze that we have used extensively (Gisquet-Verrier et al. 1989, 2004; Botreau and Gisquet-Verrier 2006). This task shares a number of features with the cross-maze task but also gives some additional advantages. As the task is automated, the goal arm for one trial serves as the start arm for the following trials. As a result, strategies such as spatial and cue strategies cannot be used, restricting the available strategies to two egocentric based strategies ("turn right," "turn left") and two visual-cue strategies ("follow the light," "follow the dark"), and thus strengthening the conflict between strategies, as well as the difficulty of the task. Another advantage of the present task is that the use of the different strategies can be easily characterized and analyzed, giving clear information about the way rats behave.

In the present study, rats were consecutively tested in two different strategy switching problems, a rule-shifting and reversal learning, for which perseverating on the previous rule had different outcomes. Maintaining the initial strategy during reversal leads to no reward, while it leads to $50 \%$ of reinforced trials, during rule shifting. Comparing the behavior of the same rats during these two changes can thus provide interesting information for specifying the respective role of PL and IL areas in the perseverative tendencies.

In the first experiment (see Fig. 2), rats with selective and combined PL and IL lesions were first trained with the Light strategy, then with their nonpreferred response strategy (ruleshifting), and finally with their preferred response (reversal learning). The second experiment was designed to determine how the order of the strategy switching, as well as the number of strategy switches, may alter the effects of lesions. To this end (see Fig. 3), control rats and rats with PL lesions were first trained with their nonpreferred response strategy, followed by their preferred response strategy (reversal learning) and then to the Light strategy (rule-shifting). These first two switches were then followed by a second rule-shifting (nonpreferred response) and a second reversal learning (preferred response). In all, the present series of experiments allows us to clarify the influence of the extent of the lesion, the order of the strategy switching, the difficulty of task, and the level of conflict, all factors that have largely been ignored but might be of critical importance (Bussey et al. 1997; Li and Shao 1998; Floresco et al. 2008).

\section{Results}

The results from this series of experiments are largely based on analysis of the rats' behavior throughout an entire strategy switching session in our new automated Y-maze task (see Materials and Methods section for explanations of the behavioral analysis conducted on the rats' performance). Strategy switching involves constituent processes, such as detection of change, inhibition of the previous strategy, testing other strategies, selecting a new correct strategy, and finally, maintaining this correct strategy. Each of these parameters will be analyzed.

\section{Histology}

Examination of the histological stained sections was made according to the Paxinos and Watson atlas (Paxinos and Watson 1986). Figure 1 shows a reconstruction of the extent of lesions for the first and the second experiment. In most cases, lesions were characterized by a central cavity (in the vicinity of injection sites), surrounded by nonfunctional necrotic tissue, composed of glial cells and picnotic-shaped neurons. Animals with unilateral cell loss or with less than $60 \%$ tissue damage of the target brain region were discarded from behavioral analyses. Only rats with global sparing of dorsal peduncular cortex (DP), anterior cingulate cortex, dorsal (ACd) and ventral (ACv), were included. Some rats with IL lesions also presented minor damage to medial orbital cortex (MO). In all, in Experiment 1, there were 13 rats (PL: 4, IL: 5, and PL/IL: 4) discarded from the behavioral analyses and three PL-lesioned rats from Experiment 2.

\section{Experiment 1}

Behavioral analyses using our new automated task were performed on rats with PL lesions $(n=13)$, IL lesions $(n=7)$, and combined PL/IL lesions $(n=11)$; with corresponding controls consisting of Sham-PL $(n=11)$, Sham-IL rats $(n=10)$, and Sham PL/IL rats $(n=10)$. As the different control groups showed no behavioral difference during the different stages of the experiment, they have been pooled in one sham-operated group comprising 31 rats. 
PL

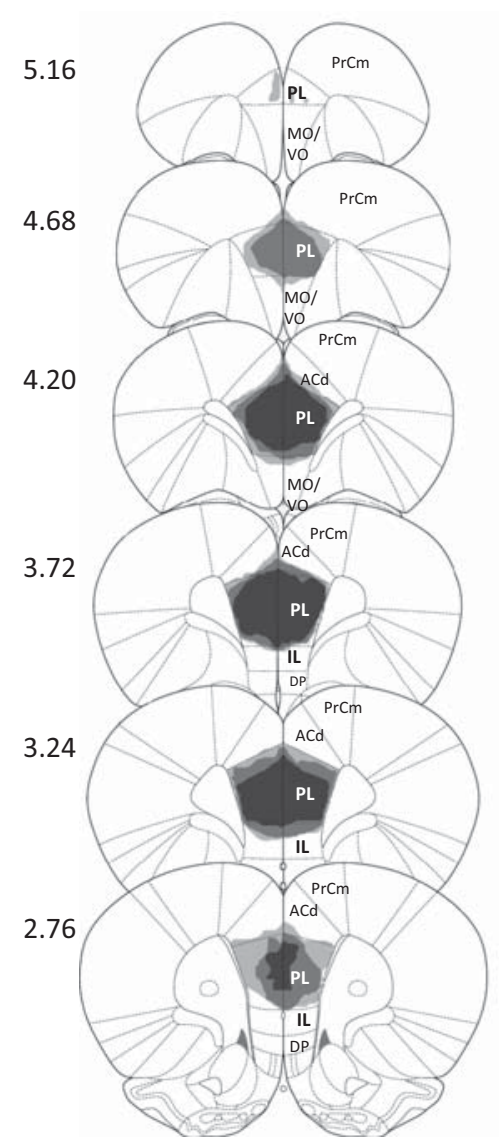

IL

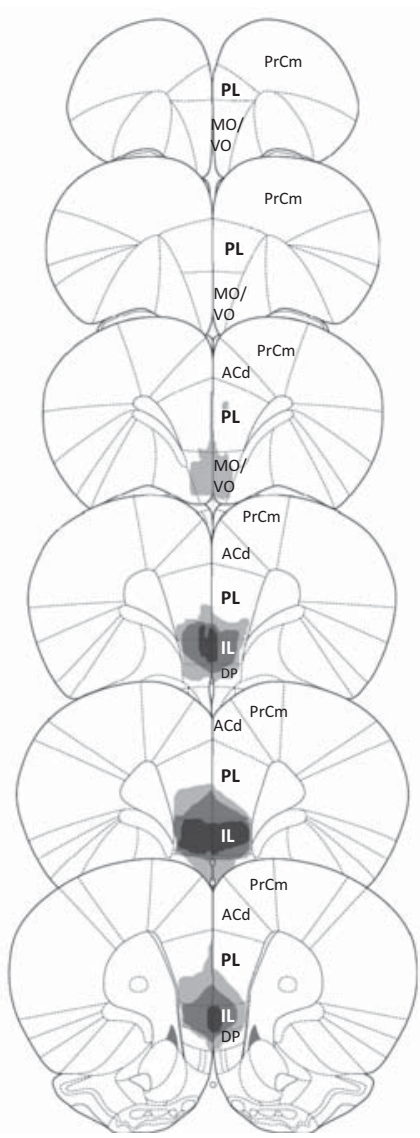

$\mathrm{PL} / \mathrm{IL}$

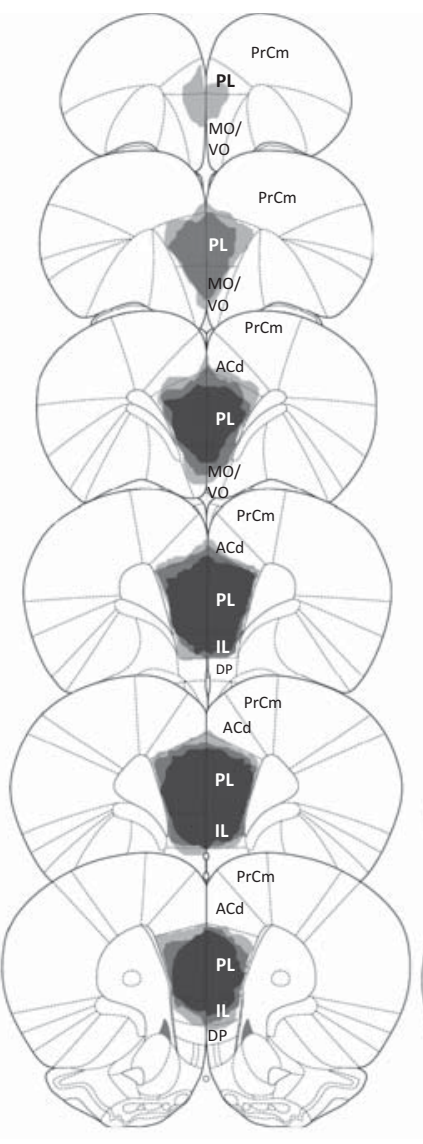

EXP2-PL

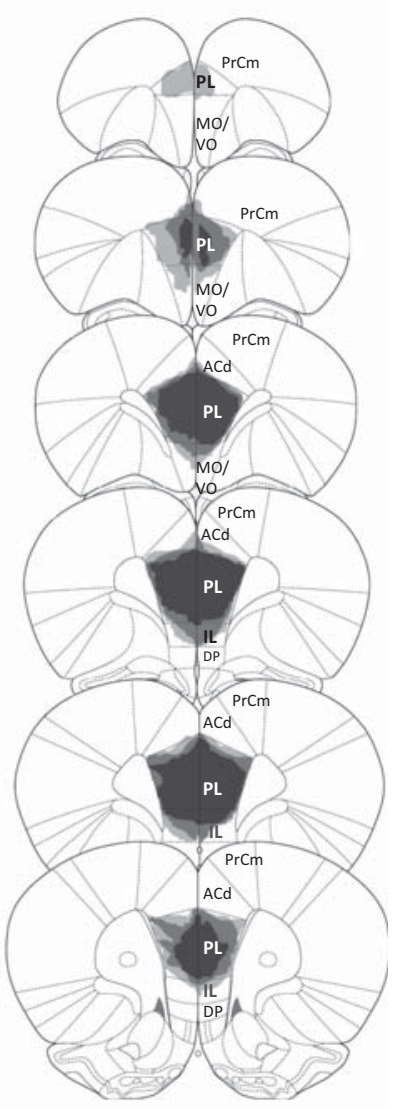

Figure 1. Reconstruction of the extent of $\mathrm{PL} / \mathrm{IL}, \mathrm{PL}$, and IL lesions in Experiment 1 , and $\mathrm{PL}$ lesions in Experiment 2 , at different rostrocaudal levels $(+5.16$ to 2.76 from bregma). Black area, indicates the region lesioned for $75 \%-100 \%$ of the rats; the gray area, for $50 \%-75 \%$, and pale gray for $25 \%-50 \%$ of the rats. Nomenclature is adapted from Krettek and Price (1977), ACd: dorsal anterior cingulated cortex, DP: dorsal peduncular cortex, IL: infralimbic cortex, MO/VO: medial orbital and ventral orbital cortices, PrCm: medial precentral cortex, PL: prelimbic cortex.

\section{Initial training: Light}

Rats were first trained to enter the lit arm in order to get a food reward. Under these conditions, rats were never reinforced when entering the dark arm, and were reinforced only half of the trials when they followed a response strategy (right or left turns).

During the initial acquisition of the Light strategy (Fig. 4A), rats first tried their naturally preferred strategy, a response strategy providing $50 \%$ of reinforced trials and spent more than $50 \%$ of their trials (Fig. 4) using this strategy, herein after referred to as the preferred response (PR). The criterion of six consecutive correct responses was reached after approximately 60 trials, with no between group differences $(F<1)$, indicating that selecting the correct strategy was not affected by lesions. Rats progressively improved their performance by remaining on the correct strategy until they reached CRI 10. It required about a hundred trials to achieve the 10 consecutive correct responses criterion. Analysis of varniance (ANOVA) performed on this data revealed no group effect $(F<1)$, nor interaction between lesion and criterion $(F<1)$.

\section{Rule-shifting: Light $\rightarrow$ NPR}

During the rule-shift strategy, rats were required to choose the nonpreferred response (NPR) strategy in order to get a food reward. During this session, the Light strategy, which was previously reinforced $100 \%$ was now reinforced 50\%, as was the dark strategy, while the PR strategy was not reinforced at all. Under these conditions, control rats needed 120 trials to reach the training criterion of 10 consecutive correct responses (Fig. 4B), a number of trials which did not significantly differ from that required during initial acquisition $\left(F_{(1,60)}=2.6\right.$, ns). Rule-shifting, however, required a significantly greater number of trials than initial training for PL/IL $\left(F_{(1,20)}=5.4, P=0.029\right)$ and PL $\left(F_{(1,24)}=12.7, P=\right.$ $0.002)$ - lesioned rats, but not for the IL-lesioned rats $\left(F_{(1,12)}=3.1\right.$, $P=0.10)$.

During the rule shifting, rats were required to choose the nonpreferred response (NPR) strategy in order to get a food reward. During this session, the Light strategy, which was previously reinforced $100 \%$ was now reinforced $50 \%$, as was the dark strategy, while the PR strategy was not reinforced at all. Under these conditions, control rats needed 120 trials to reach the training criterion of 10 consecutive correct responses (Fig. 4B), a number of trials which did not significantly differ from that required during initial acquisition $\left(F_{(1,60)}=2.6\right.$, ns). Rule shifting, however, required a significantly greater number of trials than initial training for PL/IL $\left(F_{(1,20)}=5.4, P=0.029\right)$ and PL $\left(F_{(1,24)}=12.7\right.$, $P=0.002)$-lesioned rats, but not for the IL-lesioned rats $\left(F_{(1,12)}=\right.$ $3.1, P=0.10)$.

During rule shifting (Fig. 4B), rats with PL lesions were disrupted only on CRI $10\left(F_{(1,42)}=5.3, P[\mathrm{~F}]=0.026\right)$. As indicated 
Initial training : Light
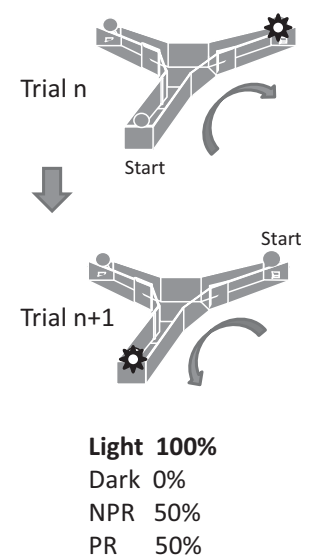

Rule-shifting: NPR
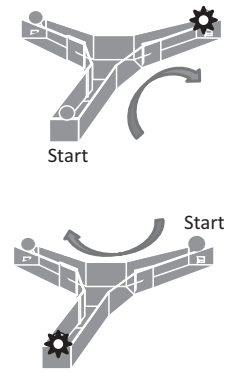

Light $50 \%$

Dark $50 \%$

NPR $100 \%$

PR $0 \%$
Reversal: PR
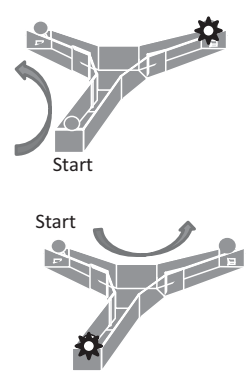

Light $50 \%$

Dark $50 \%$

NPR $0 \%$

PR $100 \%$
Figure 2. Experimental paradigm adopted for Experiment 1. Rats were trained with the Light strategy during initial training, then with their nonpreferred response (NPR) during rule-shifting, and finally, with their preferred response during reversal. Note that in every stage of the task, each strategy was associated with a particular reward contingency varying from $0 \%$ to $100 \%$.

in Figure 4B, rats with PL lesions behave as control rats during the beginning of rule shifting, but required a significantly larger number of trials than control rats to progress from CRI 6 to the end of training $\left(F_{(1,42)}=5.9, P=0.019\right)$. Rats with IL lesions required more trials than the control rats to reach CRI5 and 6 (see Fig. $4 \mathrm{~B})$, but the difference fell just short of significance $\left(F_{(1,36)}=\right.$ 3.7, $P=0.06$ ). Rats with IL lesions required a higher number of training trials than the controls to reach CRI6 from CRI3 $\left(F_{(1,36)}=3.4, P=0.069\right)$, but not during the second part of training. Rats with PL/IL lesions exhibited severe difficulties in reaching the different criteria $\left(F_{(1,40)}=4.8, P=0.033\right)$, and more specifically required more trials to reach CRI5, CRI6, CRI7, and CRI8 $\left(F_{(1,40)}=4.5, P=0.038 ; F=8.5, P=0.006 ; F=4.9, P=\right.$ 0.031 , and $F=4.3, P=0.042$, respectively). Rats with $\mathrm{PL} / \mathrm{IL}$ lesions exhibited results close to those obtained for rats with lesions to the IL, requiring significantly more training trials during the first part of rule-shifting, from CRI3 to CRI6 $\left(F_{(1,40)}=\right.$ $8.1, P=0.007)$, while behaving normally during the second part, from CRI6 to CRI10. These results suggest that $\mathrm{mPFC}$ lesions disrupted the processes required to successfully perform a rule-shifting based on the shift between a visual and a response dimension.

During rule shifting, control rats started by adopting the Light and PR strategies (i.e., the previous and their preferred strategies). As the PR strategy was no longer reinforced, rats rapidly dropped this strategy and adopted the previously correct Light strategy, which was still effective for $50 \%$ of the trials. Control rats used the Light strategy for nearly $60 \%$ of their trials (maximum average of 30 trials). As indicated in Figure 5, neither these parameters nor the use of the different strategies were significantly modified by the lesions. Seventy percent to $80 \%$ of the rats with $\mathrm{PL} / \mathrm{IL}$ and IL lesions required more than 100 trials to reach CRI6 compared with
$20 \%$ for the control rats, confirming our previous observations that these lesioned rats showed some delay in selecting the new correct strategy. Rats with lesions to the PL had no difficulty in reaching CRI 6 but required more training trials to reach the final training criterion. Indeed, PL-lesioned rats used the light strategy significantly more times than control rats during the second part of training (Cri6-10) $\left(F_{(1,42)}=9.5, P=0.004\right)$, indicating either some perseverative tendencies on the previously adopted strategy, or some difficulties to maintain their choice on the new correct strategy.

\section{Reversal: $N P R \rightarrow P R$}

On the following day, rats had to adopt the PR strategy that was not reinforced the day before. At the same time, both Light and Dark strategies were reinforced for half of the trials. Under these conditions, the number of trials necessary to reach a criterion of 10 consecutive correct responses (see Fig. 4C) was significantly higher than that required during the initial training phase (sham: $F_{(1,56)}=10.0, P=0.003$; PL: $F_{(1,20)}=14.7, P=0.001$; IL: $F_{(1,12)}=4.1, P=0.063$; PL/IL: $\left.F_{(1,16)}=13.7, P=0.002\right)$.

Analyses of the number of trials necessary to reach the different training criteria showed an effect of the lesions $\left(F_{(1,46)}=4.8\right.$, $P=0.032$ ). The main difficulties demonstrated by rats with PL lesions was between CRI4 to CRI6, PL rats requiring a significantly higher number of trials then the control rats during the first part of training, from CRI3 to CRI6 $\left(F_{(1,34)}=4.5, P=0.040\right)$. Due to the cumulative nature of our index, the difference between the PL-lesioned group and the controls only reached a significant level at CRI10 $\left(F_{(1,34)}=4.2, P=0.047\right)$. Rats with combined PL/ IL lesions exhibited results relatively close to those obtained for PL-lesioned rats, with difficulties occurring between CRI 5 to CRI 7 (Fig. 4C), and significant differences with the controls at CRI9 and 10, due to cumulative effect $\left(F_{(1,32)}=5.9, P=0.020\right.$, and $F=5.5, P=0.025$, respectively). PL/IL-lesioned rats required more trials to perform the two stages of reversal, but the effects of the PL/IL lesions were only significant on the second part of reversal, from CRI6 to CRI10 $\left(F_{(1,32)}=6.4, P=0.016\right)$. Rats with IL lesions exhibited a different pattern of results with difficulties to reach the lowest training criteria. They required 118 trials to reach CRI3 when control rats required only an average of 59.6 trials. As a consequence, disruptions in the IL-lesioned group were obtained for CRI3 and CRI5 $\left(F_{(1,32)}=4.6, P=0.038\right.$ and $F=5.7, P=0.022$, respectively).
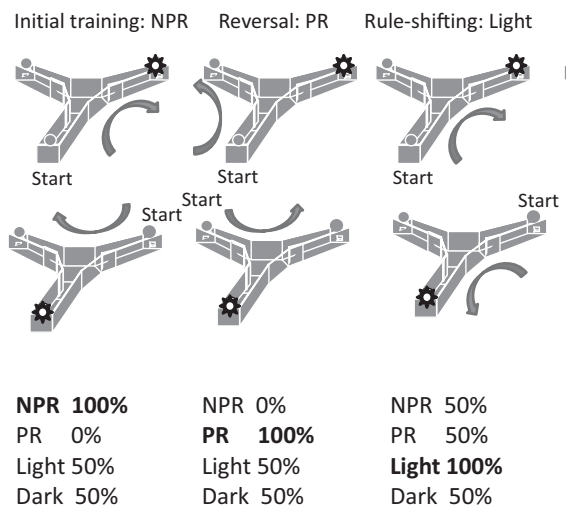

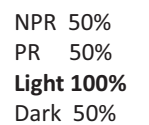

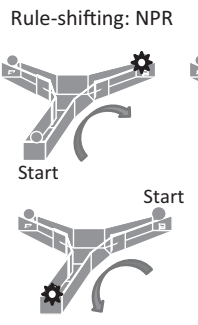

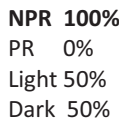

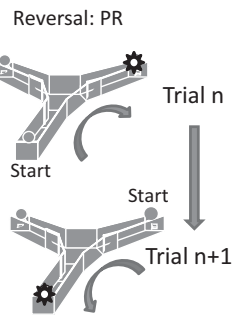

NPR $0 \%$

PR $100 \%$

Light $50 \%$

Dark $50 \%$

Figure 3. Experimental paradigm adopted for Experiment 2. Rats were trained with their nonpreferred response (NPR) during initial training, then with their preferred response during reversal, and with the Light strategy for rule-shifting. Rats were further trained again with their nonpreferred response (NPR) during a second rule-shifting, and finally, with their preferred response (PR) during a second reversal. Note that in every stage of the task, each strategy was associated with a particular reward contingency varying from $0 \%$ to $100 \%$. 
A

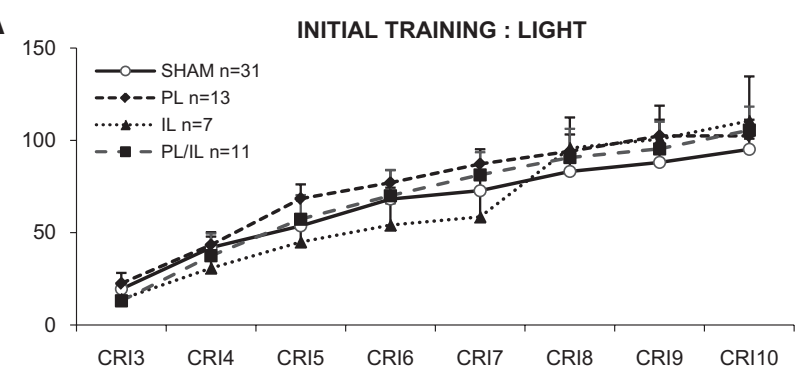

B

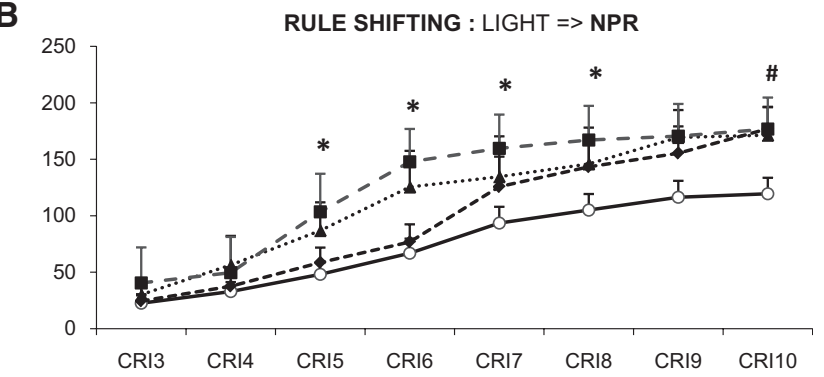

C

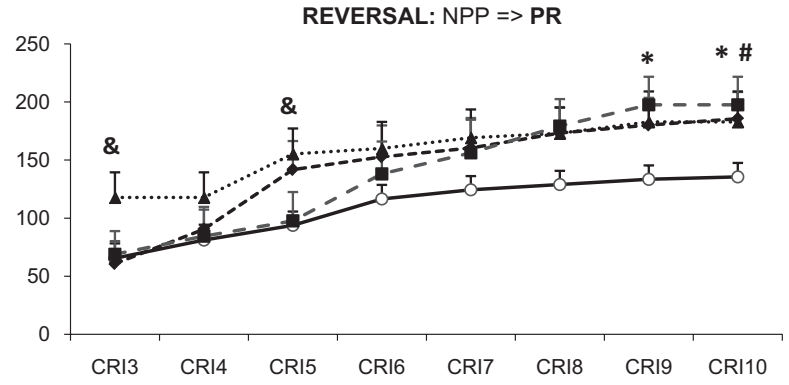

Figure 4. Mean number ( \pm SEM) of training trials necessary to reach three (CRI3) to 10 (CRI10) consecutive correct responses, in Experiment 1 during initial training $(A)$, rule shifting $(B)$, and reversal $(C)$ in shamoperated (SHAM), PL, IL, and PL/IL-lesioned rats. Significance compared to the sham-operated rats is labeled in the corresponding manner: PL-lesioned rats (\#), IL-lesioned rats (\&), and PL/IL lesioned rats $(*)$.

During reversal, rats first adopted the NPR strategy that was effective the day before. Control rats used this strategy for approximately $30 \%$ of the trials, indicating some perseverative tendencies on this now nonreinforced strategy, an effect not modified by lesions. Rats then shifted toward the Light strategy they had previously experienced and which was rewarded for $50 \%$ of the trials. They continued to use this strategy for more than $40 \%$ of their trials, with a maximum average of 20 trials (Fig. 5). Again, these effects were not modified by the lesions. Control rats tested the correct PR strategy only after 60 trials (Fig. 5) and this was prolonged in rats with IL lesions (118 trials; $\left.F_{(1,32)}=4.8, P=0.034\right)$, demonstrating difficulties in overcoming what they had previously learned. Some rats with PL and PL/IL lesions had difficulties in selecting the correct strategy, while others had difficulties in reaching the longest training criteria, indicating problems in selecting and maintaining the use of the PR strategy, without resuming the light strategy for a few trials.

\section{Conclusion of Experiment 1}

The present experiment indicates that initial training, rule shifting, and reversal are not equivalent in terms of difficulty and are not similarly affected by the lesions. Contrary to the initial training, both rule shifting and reversal were disrupted by mPFC lesions. The results from the first experiment, summarized in Table 1, indicate that control rats exhibited strong perseverative tendencies during strategy switching. During each stage, rats made extensive use of a particular strategy associated with 50\% of the reinforced trials. This strategy was either the preferred strategy, during the initial training, the previously learned strategy, during rule shifting, or another strategy previously experienced, such as the Light strategy during reversal. Careful examination of the data indicated that lesions had no discernible effects on the rats' behavior and did not greatly alter the use of the different strategies. Lesions had no effect on the rats' ability to detect when strategy switching occurred or to select other strategies. From our data, it appears that rats with IL lesions (1) required more trials than control rats to select the correct strategy during rule-shifting and (2) significantly more trials to test and select the correct strategy during reversal. Both these results indicate that disruption due to IL lesions is mainly associated with the early stages of strategy switching when they have to avoid strategies they had learned before. In contrast, disruption induced by PL lesions during ruleshifting, resulted in the rats inability to maintain the correct strategy for a large number of consecutive trials, while during reversal, difficulties were associated with selecting and maintaining the correct strategy. Rats with combined PL/IL lesions showed greater disruption than those with separate lesions. During rule shifting, rats with combined lesions had difficulties in selecting the new strategy, as rats with IL lesions, while during reversal, their disruption was closer than those of the PL lesioned rats as they had difficulties in maintaining their choice on the correct strategy.

\section{Experiment 2}

The aim of Experiment 2 was to determine whether the order of strategy switching may modulate the effects of mPFC lesions and (2) whether these effects maintain with repetitive strategy switching. To this end, we decided to study PL-lesioned rats, which exhibited larger disruptions than IL-lesioned rats, to compare their behavior with sham-operated rats when trained with successive strategies (Fig. 3). Rats were first trained (see Fig. 3) on their nonpreferred response (NPR) (initial training), their preferred response (PR; reversal 1), and then the Light strategy (ruleshifting 1). Following the first two strategy switches, rats were further trained on their NPR strategy (rule-shifting 2), and this was followed by their PR strategy (reversal 2). Behavioral analyses were performed on nine sham-operated rats and 11 rats with PL lesions.

\section{Initial training: NPR}

Rats were first trained to follow the NPR strategy during initial acquisition. Under these conditions, the PR strategy was not reinforced, while the two visual-cue strategies were reinforced for half of the trials. As illustrated in Figure 6A, rats reached the different training criteria progressively and required about one hundred trials to make 10 consecutive correct choices, i.e., approximately the same number of trials as they did during acquisition of the Light strategy in Experiment 1. Unexpectedly, however, during initial training, rats with PL lesions showed a transient facilitation in performance during the early stages of initial training; particularly at CRI4 and $5\left(F_{(1,18)}=4.6, P=0.045\right.$ and $F=10.2, P=0.005$, respectively); however, there was no interaction between development of the criterion and lesions $(F<1)$.

Rats began to use the PR and the Dark strategies, and then the NPR strategy, while largely ignoring the Light strategy. The use of the different strategies was not modified by the PL lesions $(F<1)$. Even though the PR strategy was never reinforced, rats used it quite extensively (about 35\% of their trials), with a maximum 

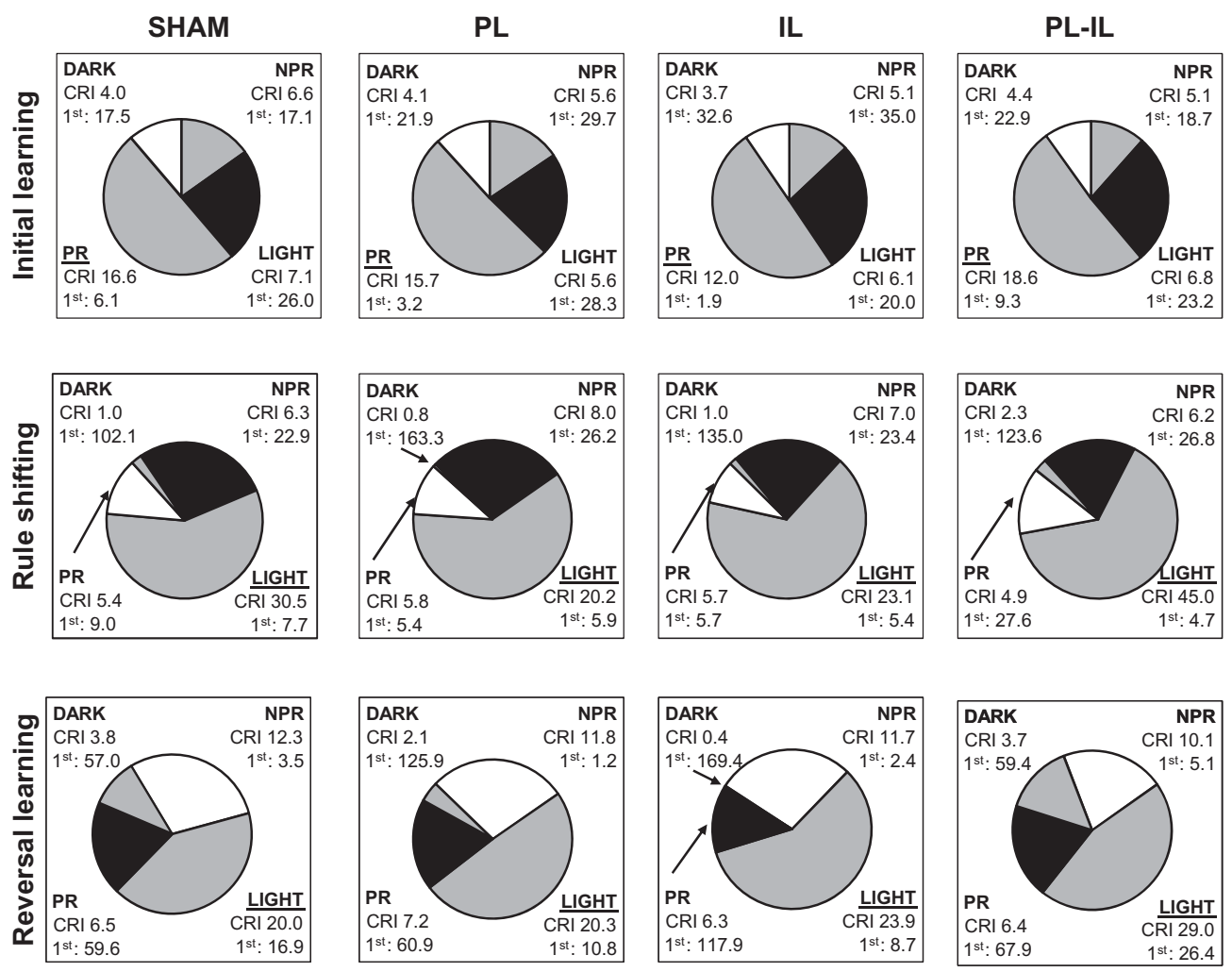

Figure 5. Mean number of trials in the Light, Dark, preferred response (PR), and nonpreferred response (NPR) strategies during initial training, ruleshifting, and reversal learning in sham-operated (SHAM), PL-, IL-, and PL/IL-lesioned rats. The average of the maximal number of trials using a particular strategy (CRI) and the number of trials before the first attempt to use this strategy (1st) are also indicated. The 100\% reinforced strategy is in black, the nonreinforced strategy is in white, and the two $50 \%$ reinforced strategies are in gray. The strategy used as the alternative strategy is underlined. Due to some overlap between strategies, the sum of the different strategies slightly exceeds $100 \%$ (from 101.5 to 112.1 ).

average of eight trials, while PL-lesioned rats showed a significantly lower maximum average $\left(F_{(1,18)}=5.42, P=0.030\right)$, revealing a reduction in perseveration on their spontaneously preferred strategy, that may account for their transient, but significant, facilitation occurring at CRI4 and 5. As depicted on Figure 7, rats tended to perseverate on the 50\% reinforced Dark strategy before progressively shifting to the NPR strategy, and this was not affected by PL lesions.

\section{Reversal: $N P R \rightarrow P R$}

The following day, rats had to reverse their choice, to select the PR strategy. During reversal, the previously rewarded NPR strategy was no longer reinforced but the two visual-cue strategies were still reinforced for $50 \%$ of the trials. As illustrated in Figure 6B, reversal was particularly difficult, as the number of trials necessary for rats to reach CRI10 was significantly higher than that required during the initial training (sham: $F_{(1,8)}=14.1, P=0.006$; PL: $\left.F_{(1,10)}=22.4, P<0.001\right)$. ANOVA conducted on the number of trials required for the different training criteria indicated a significant main effect of Lesion $\left(F_{(1,18)}=4.8, P=0.039\right)$. Disruption due to PL lesions began at CRI6 with significant differences from CRI7 to $10\left(F_{(1,18)}=4.4, P=0.048 ; F=5.9, P=0.025 ; F=\right.$ $16.5, P=0.001$; and $F=11.8, P=0.003$, respectively). The significant disruption due to PL lesions was due to nonsignificant difficulties arising for some rats during the first part of reversal to select the correct strategy and for some other rats to difficulties in the second part of reversal, to maintain that strategy (see Table 2).

During reversal, rats first used the previously correct NPR strategy, but did not maintain it for very long as it was no longer reinforced. Instead, they used the Dark strategy, which was still reinforced for half of the trials. Both groups of rats demonstrated perseverative tendencies on this strategy, but lesioned rats demonstrated exaggerated perseverative tendency, using the Dark strategy during a maximal length of 65 consecutive trials, instead of 20 in control rats $\left(F_{(1,18)}=9.2, P=0.007\right.$; Fig. 7$)$. Both groups of rats tested other strategies and were both able to switch to the new correct strategy (with no difference on the number of trials required for CRI6). Difficulties for PL lesioned rats occurred either during the first part of reversal revealing difficulties in testing the new correct strategy or during the last part of reversal, when they had difficulties in maintaining their response to the novel strategy.

\section{Rule shifting: $P R \rightarrow$ Light}

Rule shifting took place the day after reversal. Rats were now trained to follow the Light, a strategy which most of them had no prior experience of. Now the Dark strategy was not reinforced while the two response strategies were reinforced for $50 \%$ of the trials. Control rats required approximately 90 trials to reach the ruleshifting criterion, i.e., significantly less than for reversal $\left(F_{(1,8)}=\right.$ 26.4, $P<0.001)$ and this was also the case for PL-lesioned rats $\left(F_{(1,10)}=78.8, P<0.001\right)$. As illustrated in Figure 6C, the trial to criteria curves are rather flat, indicating that once tested, the correct strategy was largely selected and maintained over successive criteria. Lesioned rats required slightly more trials to reach the different criteria $\left(F_{(1,18)}=2.0, \mathrm{~ns}\right)$, and both groups of rats differed only transiently on the number of trials necessary to reach CRI5 $\left(F_{(1,18)}=5.0, P=0.037\right)$. This transient difficulty, however, did not affect the early part of rule-shifting, as revealed in Figure 6C. 
mPFC lesions and strategy switching

Table 1. Summary of the results obtained during the different stages of strategy switching in Experiment 1 for control rats (Sham) and rats with separate or combined lesions to the prelimbic (PL) and infralimbic (IL) cortices

\begin{tabular}{|c|c|c|c|c|c|}
\hline EXP1: Light & Sham & PL & IL & $\mathrm{PL} / \mathrm{IL}$ & Concluding remark \\
\hline 1st PR strategy & $6.1 \pm 1.6$ & $3.2 \pm 0.9$ & $1.9 \pm 0.9$ & $9.3 \pm 3.8$ & Testing prefered strategy: OK \\
\hline Perseveration & 16.6 & 15.7 & 12 & 18.6 & Perseveration: OK \\
\hline 1st Light (CRI 3) & $26.0 \pm 3.5$ & $28.3 \pm 5.7$ & $20 \pm 6.7$ & $23.2 \pm 6.8$ & Testing correct strategy: OK \\
\hline $\begin{array}{l}\text { CRI } 3 \rightarrow 6 \\
\text { CRI } 3 \text { to } 6\end{array}$ & $48.8 \pm 6.7$ & $54.5 \pm 8.0$ & $40.1 \pm 13.0$ & $56.4 \pm 32.5$ & Selecting of correct strategy: OK \\
\hline $\begin{array}{l}\text { CRI } 6 \rightarrow 10 \\
\text { CRI } 6 \text { to } 10\end{array}$ & $26.9 \pm 4.1$ & $25.4 \pm 10.0$ & $56.4 \pm 32.5$ & $35.5 \pm 8.4$ & Maintaining correct strategy: OK \\
\hline Light $\rightarrow$ NPR & Sham & PL & IL & $\mathrm{PL} / \mathrm{IL}$ & Concluding remark \\
\hline 1st Light & $7.7 \pm 1.0$ & $5.9 \pm 1.3$ & $5.4 \pm 1.5$ & $4.7 \pm 1.0$ & Retention of previous strategy: OK \\
\hline Perseveration & 30.5 & 20.2 & 23.1 & 45 & Perseveration: OK \\
\hline 1st NPR (CRI 3) & $22.9 \pm 7.0$ & $26.2 \pm 6.6$ & $23.4 \pm 8.2$ & $26.8 \pm 18.1$ & Testing correct strategy: OK \\
\hline $\begin{array}{l}\text { CRI } 3 \rightarrow 6 \\
\text { CRI } 3 \text { to } 6\end{array}$ & $44.3 \pm 11.2$ & $48.8 \pm 14.8$ & $\begin{array}{l}95.3 \pm 30.6 \\
(\text { cri 6) }\end{array}$ & $\begin{array}{l}(107.3 \pm 19.4)^{*} \\
\text { cri } 5.6\end{array}$ & Selecting correct strategy: IL PL/IL \\
\hline $\begin{array}{l}\text { CRI } 6 \rightarrow 10 \\
\text { CRI } 6 \text { to } 10\end{array}$ & $52.7 \pm 11.0$ & $\begin{array}{l}100.2 \pm 14.8 \\
\text { cri } 10\end{array}$ & $45.9 \pm 16.9$ & $\begin{array}{l}29.0 \pm 7.6 \\
\text { cri } 7,8,(9,10)^{*}\end{array}$ & Maintaining correct strategy: PL PL/IL \\
\hline $\mathrm{NPR} \rightarrow \mathrm{PR}$ & Sham & PL & IL & $\mathrm{PL} / \mathrm{IL}$ & Concluding remark \\
\hline 1 st NPR & $3.5 \pm 1.2$ & $1.2 \pm 0.6$ & $2.4 \pm 0.7$ & $5.1 \pm 3.8$ & Retention of previous strategy: OK \\
\hline Perseveration & 12.3 & 11.8 & 11.7 & 10.1 & Perseveration: OK \\
\hline 1st Light (50\%) & $16.9 \pm 3.9$ & $10.8 \pm 3.2$ & $8.7 \pm 3.7$ & $26.4 \pm 11.2$ & Testing alternative strategy: OK \\
\hline Perseveration & 20 & 20.3 & 23.9 & 29 & Perseveration: OK \\
\hline 1st PR (CRI 3) & $60.1 \pm 12.4$ & $60.9 \pm 19.4$ & $117.9 \pm 21.6$ & $69 \pm 19.9$ & Testing correct strategy: IL \\
\hline $\begin{array}{l}\text { CRI } 3 \rightarrow 6 \\
\text { CRI } 3 \text { to } 6\end{array}$ & $56.6 \pm 9.8$ & $(91.9 \pm 18.7)^{*}$ & $\begin{array}{l}42.1 \pm 14.1 \\
\text { cri } 3,5\end{array}$ & $69.1 \pm 24.3$ & Selecting correct strategy: IL PL \\
\hline $\begin{array}{l}\text { CRI } 6 \rightarrow 10 \\
\text { CRI } 6 \text { to } 10\end{array}$ & $19.0 \pm 4.7$ & $\begin{array}{l}32.9 \pm 10.3 \\
\text { cri } 10\end{array}$ & $22.9 \pm 9.8$ & $\begin{array}{l}59.4 \pm 26.8 \\
\text { cri } 9,10\end{array}$ & Maintaining correct strategy: PL/IL PL \\
\hline
\end{tabular}

Different parameters were considered: the number of trials required before the first use of a particular strategy (1st), the maximal number of trials using a particular strategy (CRI), which constitutes an index of perseveration, the number of trials required to reach CRI 6 from CRI 3 (CRI $3 \rightarrow 6$ ) or CRI 10 from CRI 6 (CRI $6 \rightarrow 10$ ), and the different criteria for which individual differences have been obtained.

During rule shifting, rats in both groups rapidly dropped the now nonreinforced Dark strategy and selected the PR strategy used the previous day, which was now reinforced for half of the trials. Both groups of rats developed strong perseverative tendencies on the PR strategy as they used this strategy for approximately $80 \%$ of the trials (Fig. 7). Rats with PL lesions exhibited a maximum average of 45 trials using that strategy, which was significantly higher than for the controls $\left(F_{(1,18)}=4.5, P=0.046\right)$, indicating either additional perseverative tendencies on the partially reinforced PR strategy or difficulties in selecting the new Light strategy. The first attempt to use the 100\% reinforced Light strategy occurred after 57 trials with control rats and 77 with lesioned rats, but the difference did not reach a significant level $\left(F_{(1,18)}=\right.$ $2.3, P=0.144)$. Disruption due to PL lesions mainly occurred during the first part of training. Lesioned rats perseverated on this strategy during a maximal number of 46 consecutive trials, instead of 29 in control rats (Fig. 7). However, once lesioned rats determined that the Light strategy was the most effective (CRI6), they continued using this strategy, rarely went back to the PR strategy, and took less than 25 trials to reach CRI10 from CRI6, with no difference between groups.

\section{Rule shifting 2: Light $\rightarrow$ NPR}

A second rule shifting was tested the day following the first one. Rats were now required to follow the NPR strategy for continuous reinforcement. Following the previous Light or Dark strategy was reinforced during half of the trials, whereas following the PR strategy was not reinforced at all.
As illustrated in Figure 6D, rats required a mean of 126 trials to reach the training criterion, a number that was not significantly different from that required during initial training and ruleshifting ( $F<1$ and $F_{(1,8)}=4.2$, ns, respectively) but lower than that required for the first reversal $\left(F_{(1,8)}=15, P=0.004\right)$. Rats with PL lesions were only slightly disrupted when they reached the longest training criteria, but the differences were not significant $\left(F_{(1,18)}=1.5, \mathrm{~ns}\right)$ and strategies used to solve this shift were not affected either by lesions (Fig. 7).

\section{Reversal 2: $N P R \rightarrow P R$}

Finally, rats were given a second reversal, where they were required to use the PR strategy. Here, the previous NPR strategy was not reinforced, while the two response strategies were reinforced for half of the trials. As shown in Figure 6E, this second reversal was much easier to master than the first one and was achieved with only 134 trials, i.e., significantly less than the first reversal $\left(F_{(1,8)}=9.6, P=0.014\right)$. The number of trials, however, was not different from that required for the other discriminations $(F<1$, $F=4.2$, ns). During this second reversal, PL lesions did not affect the rate of acquisition but significantly decreased perseveration (Fig. 7) on the previously valid NPR strategy $\left(F_{(1,18)}=4.5\right.$, $P=0.045)$.

\section{Conclusion of Experiment 2}

The initial acquisition, reversal 1 and 2, as well as rule-shifting 1 and 2 , were not equivalent in terms of difficulty. The first 

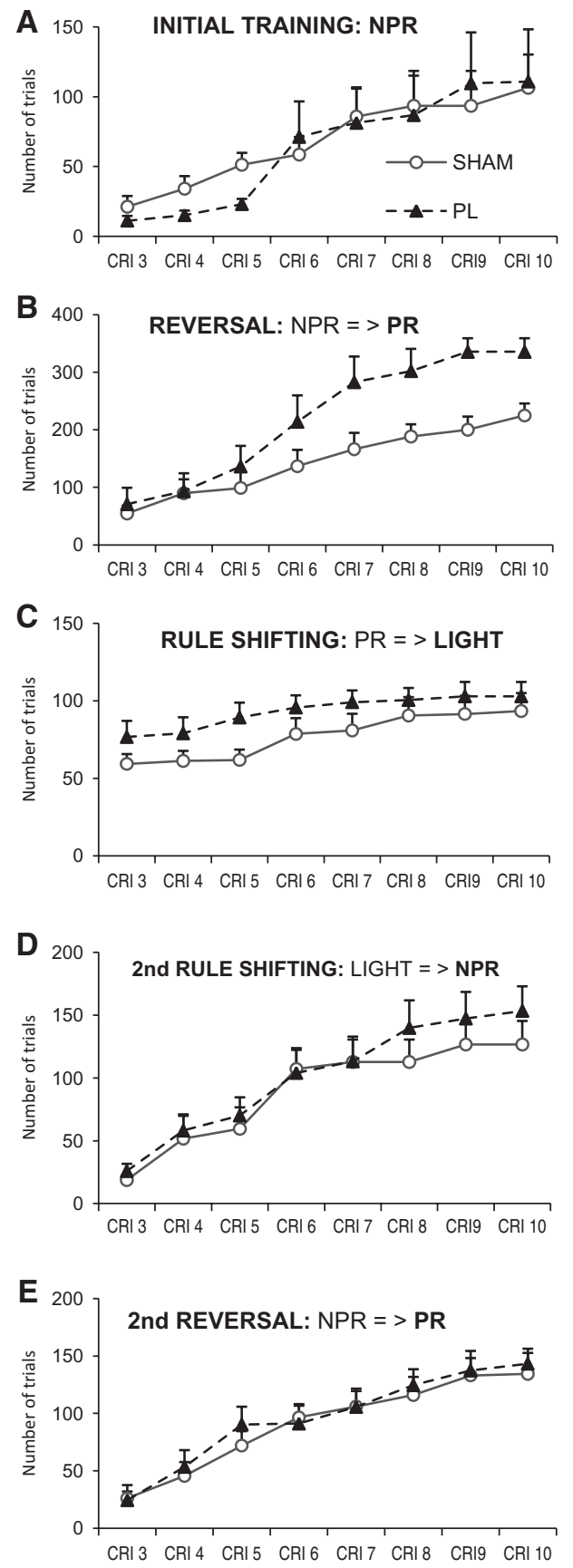

Figure 6. Mean number ( \pm SEM) of training trials required to reach three (CRI3) to 10 (CRI10) consecutive correct responses, during initial training $(A)$, first reversal $(B)$, first rule shifting $(C)$, second rule shifting $(D)$, and second reversal $(E)$ in sham-operated (SHAM) and PLlesioned rats.

reversal was attained after significantly more trials than the other switches. Results from Experiment 2 are summarized in Table 2. PL lesions slightly facilitated the early stage of the initial acquisition and largely disrupted the latest stage of reversal by strengthening the tendency for perseveration on the partially reinforced Dark strategy that was not the previously correct strategy. Thereafter, rats with PL lesions were only slightly affected by the first ruleshifting and had some difficulties in testing the new correct strategy and in selecting the correct strategy but no longer during the other strategy switches.

\section{Discussion}

The present series of experiments used a new task to investigate the involvement of the mPFC, and more specifically the role of the two main subregions, the PL and IL cortices, in strategy switching. Our new task allows analyzing rats' behavior during strategy switching. During each discrimination, rats tend to perseverate with a strategy reinforced for half of the trials. Interestingly, mPFC lesions induce rather subtle effects on rats' behavior, and in general, do not alter the relative use of the different strategies, or the way rats solved strategy switching. The different lesions had no disruptive effect on the initial discrimination but increased the number of trials necessary to achieve ruleshifting and reversal learning. We showed that disruption due to PL lesion was due to increases in perseverative tendencies, as previously proposed (Ragozzino et al. 1999a; Block et al. 2007). Our data, however, go further in indicating that (1) PL lesions only potentiated natural perseverative tendencies developed by control rats during strategy switching and (2) these exaggerated perseverative tendencies induced by PL lesions always concerned a 50\% reinforced strategy, that is, the previously learned strategy for rule-shifting and an alternative strategy for reversal learning. The present results demonstrate that rats with PL and IL lesions have no difficulty dropping out of a previous strategy when no longer effective, but exhibited difficulties to acquire the new one. As summarized in Table 3, rats with lesions to the PL mainly had a deficit in reaching the performance criteria from CRI6, suggesting therefore difficulties in selecting and maintaining the new correct strategy. Rats with IL lesions showed a different profile as their disruption principally occurred during the first part of strategy switching and resulted from difficulties in testing and selecting the new correct strategy, particularly when not relevant before. Finally, both experiments show that lesions more largely disrupted behavior during the first than the second strategy switching. No disruptive effect was obtained for rats with PL lesions during subsequent switches, indicating that after an initial test, strategy switching can be correctly processed without PL cortex.

\section{Characteristics of our automated strategy-switching task}

In our automated strategy-switching task, each discrimination was achieved after a number of trials largely higher (between 93 and 224 trials) than those generally required in other studies, even those using the cross-maze task (Birrell and Brown 2000; Rich and Shapiro 2007; Floresco et al. 2008; Tait et al. 2009). Several aspects contributed to the task difficulty. One is that rats were principally trained using strategies that opposed their natural tendencies, such as the Light and NPR strategies. Another aspect is that our task places a heavy emphasis on response conflict, referring as actual or perceived opposition of responses. In our task, during a particular discrimination, each strategy is associated with a specific reward contingency $(0 \%, 50 \%$, or $100 \%)$ and strategy switching leads to partial reorganization of these contingencies. In some situations, rats had to select a strategy that was previously either partially reinforced (rule-shifting) or not reinforced (reversal), resulting in more or less conflict between strategies which seems to have critical importance to determine the relative effects of the $\mathrm{mPFC}$ lesions.

\section{How rats solve the task and how lesions alter these processes}

Analyses of our data allow us to determine the way rats solve the different stages of the task and shed light on the way mPFC lesions may affect behavioral flexibility. During the initial acquisition, 

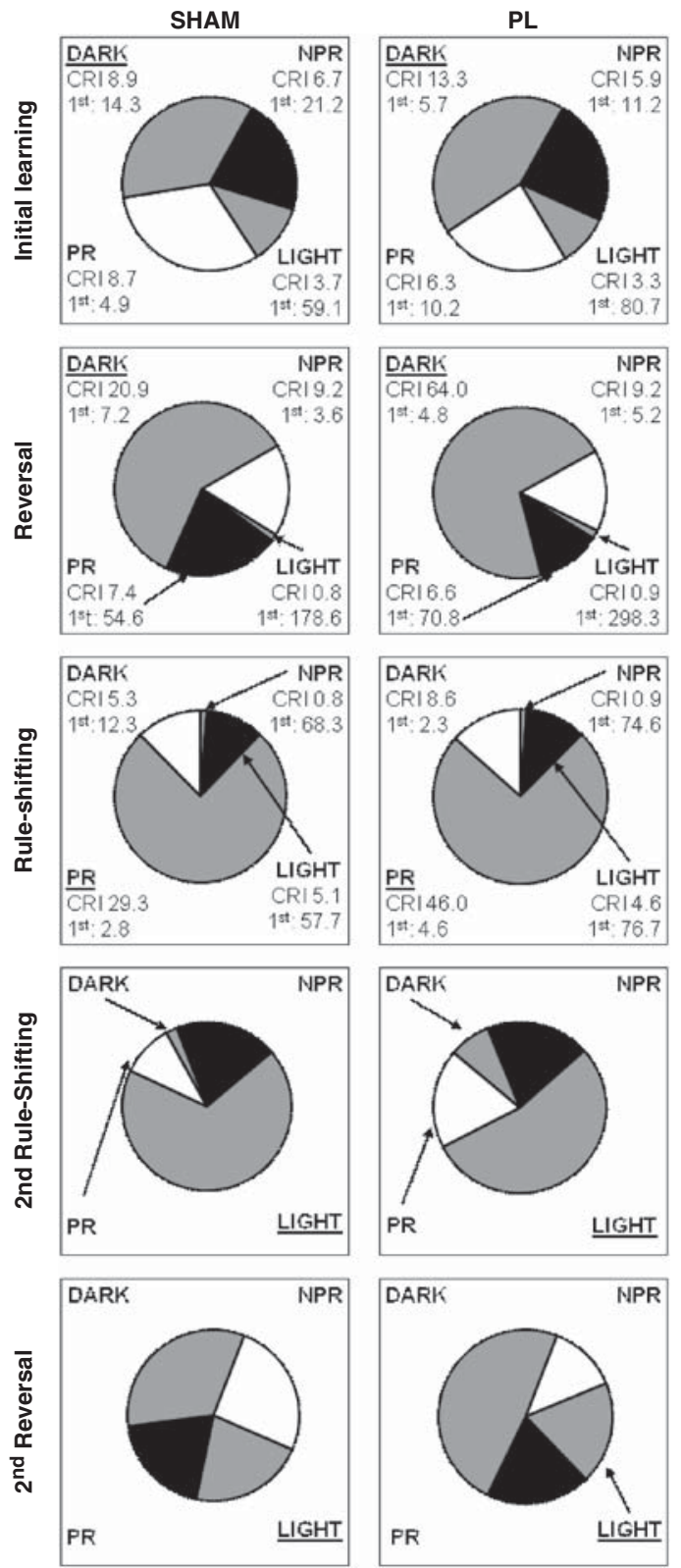

Figure 7. Mean number of trials in the Light, Dark, preferred response $(\mathrm{PR})$, and nonpreferred response (NPR) strategies during initial training, rule-shifting, and reversal learning in sham-operated (SHAM) and PL-lesioned rats. The average of the maximal number of trials using a particular strategy (CRI) and the number of trials before the first attempt to use this strategy (1st) are also indicated. The $100 \%$ reinforced strategy is in black, the nonreinforced strategy is in white, and the two strategies $50 \%$ reinforced strategies are in gray. The strategy used as the alternative strategy is underlined. Due to some overlap between strategies, the sum of the different strategies slightly exceeds $100 \%$ (from 104.7 to 111.7 ).

rats spontaneously select one of their preferred strategies that give reinforcement for $50 \%$ of the trials (PR for Experiment 1 and Dark for Experiment 2) and perseverate with this strategy for more than half of the trials, before shifting for the continuously reinforced strategy. Initial training was not affected by mPFC lesions, and in Experiment 2, rats with PL lesions were even quicker than controls to select the correct strategy because they perseverated less than control rats on their favorite strategy. Hence, when the reward contingencies are completely new, rats with lesions, as control rats, were able to shift from a spontaneously preferred, but partially reinforced strategy, to a less preferred but fully reinforced strategy. Hence, during initial training, when the level of conflict between strategies is minimal, PL and IL cortices are not required for behavioral flexibility.

During rule-shifting, rats are placed in a situation where pursuing the previous strategy led to a reinforcement for half of the trials. All rats perseverated with that strategy for more than $60 \%$ of the trials. In parallel, they progressively learned that a strategy that was previously partially reinforced (during initial training for Experiment 1, and during initial training and reversal, for Experiment 2) is now fully reinforced. This shift, which requires some behavioral flexibility, is also somewhat conflictual as rats had to choose a strategy that they previously learned not to be the correct strategy. Under these conditions, lesioned rats tended to perseverate with the previous strategy but apparently for a different purpose. Rats with IL and PL/IL lesions had difficulties in selecting the new correct strategy, while the rats with PL lesions had difficulties in selecting and maintaining their choices on the strategy. During reversal, using the previous strategy led to no reward. Under these nonconflictual conditions, control and lesioned rats were able to drop the previous strategy when it was no longer relevant, demonstrating some capacity for behavioral flexibility. Rats shifted from the previous strategy to another one they had already experienced and which was rewarded for half of the trials (Light for Experiment 1 and Dark, for Experiment 2). Interestingly, rats used their prior experience to choose an alternative strategy, and this was not altered by any of the lesions. Rats selected this alternative strategy for more than $40 \%$ of the trials, a tendency that was exacerbated in rats with lesions. Once again, rats with IL lesions exhibited difficulties in selecting the new strategy, while rats with PL and PL/IL lesions had difficulties in selecting and maintaining the correct choice on the new strategy. Here we showed that lesions increased perseveration with an alternative strategy and not with the previously correct strategy. This result demonstrates that disruption due to lesions was not due to an inability to change from a previously valid strategy, but rather an inability to select a previous strategy that was not valid. Hence, it appears that disruption resulting from mPFC lesions is not directly due to disruption in strategy switching but more likely results from difficulties to solve the response conflict generated by what they previously learned, which in turn affects strategy switching.

The present results indicate that behavioral deficits induced by PL and IL lesions were more severe in the first strategy switching rather than on the second, regardless of their order. Rats with PL lesions showed no impairment with subsequent changes, although they still required more than one hundred trials to reach criterion for switching their strategy. These results suggest that rats did not acquire a learning set enabling them to switch strategy more rapidly. One may rather suggest that increasing the number of strategy switches increased the number of reward contingencies associated with each strategy, and thus decreased the response conflict, resulting in less performance disruption.

\section{Coherence between the present results and the literature} The present results indicate that the mPFC is not involved in the resolution of discrimination, even when the discrimination is difficult to solve. This finding is in agreement with most previous studies showing the mPFC is not required to acquire a task, even when difficult (Bussey et al. 1997; Li and Shao 1998; Floresco et al. 2008). The present experiments further indicate that the mPFC lesions disrupt strategy switching, confirming previous results (Ragozzino et al. 1999b). With our automated task, we 
Table 2. Summary of the results obtained during the different stages of strategy switching in Experiment 2 for control rats (Sham) and for rats with lesions of the prelimbic (PL) cortex

\begin{tabular}{|c|c|c|c|}
\hline EXP2: NPR & Sham & PL & Concluding remark \\
\hline 1st Choice PR & $4.9 \pm 2.4$ & $10.2 \pm 4.7$ & Testing the prefered strategy: OK \\
\hline Perseveration & 8.7 & 6.3 & Perseveration: OK \\
\hline 1st NPR (CRI 3) & $21.2 \pm 7.7$ & $11.2 \pm 3.4$ & Testing the correct strategy: OK \\
\hline $\begin{array}{l}\text { CRI } 3 \rightarrow 6 \\
\text { CRI } 3 \text { to } 6\end{array}$ & $37.3 \pm 13.4$ & $\begin{array}{l}60.0 \pm 26.9 \\
\text { cri4 } 45\end{array}$ & Selecting of the correct strategy: $\mathrm{PL}+$ \\
\hline $\begin{array}{l}\text { CRI } 6 \rightarrow 10 \\
\text { CRI } 6 \text { to } 10\end{array}$ & $47.8 \pm 24.0$ & $39.8 \pm 14.0$ & Maintaining the correct strategy: OK \\
\hline $\mathrm{NPR} \rightarrow \mathrm{PR}$ & Sham & PL & Concluding remark \\
\hline 1st NPR & $3.6 \pm 1.7$ & $5.2 \pm 1.8$ & Retention of the previous strategy: OK \\
\hline Perseveration & 9.2 & 9.2 & Perseveration: OK \\
\hline 1st Dark (50\%) & $7.2 \pm 1.7$ & $4.8 \pm 1.9$ & Testing the alternative strategy: OK \\
\hline Perseveration & 20.9 & 65 & Perseveration: enhanced for $\mathrm{PL}$ \\
\hline 1st PR (CRI3) & $54.6 \pm 13.7$ & $70.8 \pm 28.5$ & Testing the correct strategy: OK \\
\hline $\begin{array}{l}\text { CRI } 3 \rightarrow 6 \\
\text { CRI } 3 \text { to } 6\end{array}$ & $90.7 \pm 25.1$ & $143.2 \pm 39.2$ & Selecting the correct strategy: OK \\
\hline $\begin{array}{l}\text { CRI } 6 \rightarrow 10 \\
\text { CRI } 6 \text { to } 10\end{array}$ & $88.1 \pm 23.9$ & $\begin{array}{l}121.5 \pm 39.9 \\
\text { cri } 7,8,9,10\end{array}$ & Maintaining the correct strategy: PL \\
\hline $\mathrm{PR} \rightarrow \mathrm{LIGHT}$ & Sham & PL & Concluding remark \\
\hline 1st PR & $2.8 \pm 0.9$ & $4.6 \pm 1.1$ & Retention of the previous strategy: OK \\
\hline Perseveration & 29.3 & $(46)^{*}$ & Perseveration: Slightly enhanced for $\mathrm{PL}$ \\
\hline 1st Light (CRI 3) & $57.7 \pm 5.6$ & $76.7 \pm 10.4$ & Testing the correct strategy: OK \\
\hline $\begin{array}{l}\text { CRI } 3 \rightarrow 6 \\
\text { CRI } 6 \text { to } 10\end{array}$ & $19.3 \pm 10.9$ & $\begin{array}{l}19.0 \pm 7.0 \\
\text { cri5 }\end{array}$ & Selecting the correct strategy: PL \\
\hline $\begin{array}{l}\text { CRI } 6 \rightarrow 10 \\
\text { CRI } 6 \text { to } 10\end{array}$ & $14.8 \pm 6.6$ & $7.2 \pm 3.1$ & Maintaining correct strategy OK \\
\hline
\end{tabular}

Different parameters were considered: the number of trials required before the first use of a particular strategy $(1 \mathrm{st})$, the maximal number of trials using a particular strategy (CRI), which constitutes an index of perseveration, the number of trials required to reach CRI 6 from CRI 3 (CRI $3 \rightarrow 6$ ) or CRI 10 from CRI 6 (CRI $6 \rightarrow 10)$ and the different criteria for which individual differences have been obtained.

have been able to show that the involvement of PL and IL cortices is, however, restricted to situations where rats have to acquire information that is not in agreement with what they previously learned. In addition, in our situation, disruptive effects of mPFC lesions were observed not only for rule-shifting but also for reversal, a result which stands in contrast to several results in the literature where lesions, inactivation or pharmacological manipulation of the PL/IL have been shown to impair shifts between dimensions, or rule-shifting but not reversal learning (Ragozzino et al. 1999b; Boulougouris et al. 2007; Rich and Shapiro 2007; Floresco et al. 2009). Reversal has often been considered as a simple form of behavioral flexibility that entails shifting between different stimulus-reward associations, and which relies on the integrity of the orbitofrontal cortex (McAlonan and Brown 2003; Kim and Ragozzino 2005; Floresco and GhodsSharifi 2007). On the other hand, extra-dimensional shift in an attentional-set shifting task or rule-shifting in a cross-maze task is generally considered as engaging more complex forms of behavioral flexibility and relies on the mPFC (Wise et al. 1996; Dalley et al. 2004; Ragozzino 2007). In fact, examination of the literature indicates that temporary inactivation, or targeted pharmacological manipulations centered on the rat mPFC have produced somewhat equivocal effects with reversal learning. Indeed, some studies were able to show that mPFC lesions can lead to reversal disruption (Kolb et al. 1974; Bussey et al. 1997; Li and Shao 1998; De Bruin et al. 2000; Ferry et al. 2000; Kinoshita et al. 2008) or even facilitation (Salazar et al. 2004; McDonald et al.
2007). Importantly, although less well documented, disruption of rule-shifting due to mPFC dysfunction has not always been reported (Rich and Shapiro 2007; Floresco et al. 2008; Young and Shapiro 2009). Therefore, it is probably too simplistic to consider that the involvement of mPFC is only determined by the type of strategy switching. In the studies using the standard procedure initiated by Birrell and Brown (2000; Tait et al. 2009), rats were required to dig for a food reward focusing on one dimension; for example, the bowl odor, and to ignore the two others (digging medium and texture). During reversal, the initial odor was no longer reinforced. In this two-choice situation, to stop choosing the initial odor obviously leads to choose the other odor, a process rendering reversal learning easy. Under these conditions, reversal was quickly obtained (with less than 10 trials) and not affected by mPFC lesions. When rats are required to consider a new dimension (i.e., the digging medium, for instance), this shift occurs after repeated learning to ignore it, which requires strong inhibition of their natural tendency in foraging. As a consequence, the extra-dimensional shift occurs with a great deal of response conflict, as rats have to choose a stimulus that they had learned to be irrelevant. In fact, this extra-dimensional shift has some common features with our reversal, as in both cases rats have to select a previously irrelevant strategy. Under these conditions, the number of trials required to reach the criterion was higher and further exacerbated by mPFC lesions (Birrell and Brown 2000). Accordingly, the involvement of the mPFC in strategy switching may not be determined by the type of change (shift of dimensions or reversal), but more likely by the amount of conflict generated by the training conditions. Our view is further supported by a previous study using a rotating T-maze, presenting the same characteristics as our maze with respect to the use of the goal arm for one trial and as a start arm for the next. With this protocol, it was shown that rats with both PL and IL lesions were severely impaired in reversal learning of a visual discrimination task (Li and Shao 1998).

Our findings further show that disruptions due to PL lesions tended to become weaker with subsequent switches, with no deficit after the second shift. Similar results have also been reported in rats with PL/IL inactivation during serial rule-shifting (Rich and Shapiro 2007) and rats with mPFC lesions when tested on

Table 3. Summary of the conclusion provided by Experiments 1 and 2 concerning the functional role of the prelimbic (PL), the infralimbic (IL), or both (PL/IL) cortices

\begin{tabular}{ccccc}
\hline & EXP1 & & EXP2 \\
\hline IL & PL/IL & PL & & PL \\
\hline $\begin{array}{c}\text { Testing }+ \\
\text { Selecting }\end{array}$ & $\begin{array}{c}\text { Selecting }+ \\
\text { Maintaining }\end{array}$ & $\begin{array}{c}\text { Selecting }+ \\
\text { Maintaining }\end{array}$ & & $\begin{array}{c}\text { Selecting }+ \\
\text { Maintaining }\end{array}$ \\
\hline
\end{tabular}


serial reversal learning (Kinoshita et al. 2008). As previously noted, the fact that rats still required a high number of trials to perform the latter strategy switches suggests that they did not acquire a learning set (Rich and Shapiro 2007). Accordingly, the lack of involvement of the PL during serial strategy switching more likely results from a progressive decrease in response conflict, resulting from successive changes.

\section{What is the functional role of PL and IL cortices?}

To our knowledge, although great emphasis has been placed on the role of the mPFC in strategy switching, very few studies tried to disentangle the respective role of the infralimbic and prelimbic cortex (Li and Shao 1998; Boulougouris et al. 2007; Rich and Shapiro 2009). The present results show that PL and IL disrupted rule-shifting and reversal in a differential manner. PL/IL lesions impaired strategy-switching more so than individual IL and PL lesions, suggesting some additive effect. It is noteworthy that rats with PL/IL lesions behave in a very similar manner to those with IL lesions during rule-shifting and with rats with PL lesions during reversal.

The disruptive effects of the lesions cannot be due to impairment in working memory, as PL lesions neither affected initial training nor the latest strategy switching while the working memory load was kept constant. Up to now, the deficits associated with PL or PL/IL lesions have mainly been interpreted as resulting from perseveration of a previously acquired strategy (but see Delatour and Gisquet-Verrier 1996, 1999, 2000). The present results confirmed that lesions to the PL increased perseverative tendencies.

They further indicate that (1) PL and IL lesions strengthen natural perseverative tendencies developed in control rats and (2) the perseverative tendencies do not result from difficulties in inhibiting a previously valid strategy, as generally proposed, but are more likely due to difficulties in shifting to a novel strategy, which was not the correct strategy before. This conclusion is particularly well illustrated by the data obtained during reversal. These results demonstrate that PL lesions did not affect the shift from one strategy to the other but rather the rats' ability to select and maintain the use of the new strategy (see also Block et al. 2007), particularly when rats learned before that this strategy was partially or completely ineffective.

Converging evidence implicates the infralimbic region of the mPFC in the ability to decrease fear during extinction, a process generally considered to be mediated by the formation of inhibitory associations (Quirk et al. 2000; Milad and Quirk 2002; Lebron et al. 2004; Sierra-Mercado et al. 2006). Here, rats with IL lesions did not exhibit any extinction deficit, as IL damage did not strengthen perseveration either on the previously valid strategy (during rule-shifting), confirming previous observations (Chudasama and Robbins 2003), or on the partially valid strategy (during reversal). With our task, excitotoxic IL lesions have been shown to transiently disrupt rule-shifting and reversal learning and more specifically, the first stage of strategy switching during which rats have to initiate responses to the new strategy. IL lesions particularly affected the strategy selection during reversal, i.e., when the new strategy was previously irrelevant. These findings suggest that impairment due to IL lesions may be attributable to difficulties in using a previously nonrewarded strategy. It is important to note that fear extinction and the ability to select a previously irrelevant strategy are both processes related to the capacity for inhibiting previously acquired information, which is also required to solve response conflict. Once rats selected the correct strategy (CRI6), the IL lesions no longer affected the performance. The present results are the first to propose a cognitive role for the IL cortex in line with that proposed for the PL cortex that has dense connections (Hoover and Vertes 2007).
Interestingly, the hypothesis that mPFC may be involved in the resolution of response conflict has already been formulated. It has been reported that rats with large mPFC lesions and with PL (but not IL) inactivation were unable to use contextual information to discriminate between ambiguous information (Haddon and Killcross 2006; Marquis et al. 2007). The present results are in line with these previous findings and support the notion that the PL/IL area may be more generally involved in the resolution of response conflict. In humans and primates, functional imaging and electrophysiological studies have shown that PFC is a key structure of conflict monitoring (Van Veen et al. 2001; Egner and Hirsch 2005; Wittfoth et al. 2009). The most influential model proposes a role for the anterior cingulate cortex (including Brodmann's areas 24, 25, and 32) in conflict detection, while dorsolateral PFC resolves conflict (Botvinick et al. 2001) by favoring the task relevant behavior (Carter and Van Veen 2007; Mansouri et al. 2009). While rat PL/IL cortices cannot be assimilated to primate dorsolateral PFC on the basis of anatomical data (Preuss, 1995; Delatour 2000), numerous studies proposed a functional homology between these two structures (Granon et al. 1994; Brown and Bowman 2002), a result further supported by the present data.

\section{Conclusion}

Viewed collectively, these data confirm previous evidence showing that the prelimbic and the infralimbic cortex are engaged in strategy switching tasks. We have generated data indicating that mPFC lesions do not directly affect behavioral flexibility. Rats with PL and IL lesions were able to shift from a partially rewarded spontaneous strategy to a nonnaturally preferred strategy during initial training, as well as to drop a previously relevant strategy when it is no longer rewarded, to the benefit of a partially reinforced and already known strategy, during reversal.

The present findings support the notion that PL and IL regions of the rodent $\mathrm{mPFC}$ are not directly involved in behavioral flexibility but more likely in the resolution of response conflict. The present study is one of the first experiments providing arguments that dissociate the respective roles of the PL and IL in strategy switching. Based on the present findings, we propose that lesions to the PL and IL impair different aspects of the resolution of response conflict, namely, the choice of a previously irrelevant strategy associated with the infralimbic cortex, whereas selection and maintenance of that strategy were associated with the prelimbic cortex.

The hypothesis that the PL/IL region is involved in the resolution of response conflict and could account for most of the impairments shown in the experiments illustrates its involvement in behavioral flexibility. This includes experiments using a shift between spatial and visual or egocentric strategies (Seamans et al. 1995; Ragozzino et al. 1999b; De Bruin et al. 2000), attentional set-shifting (Birrell and Brown 2000), shift between matching and nonmatching strategies (Joel et al. 1997; Dias and Aggleton 2000), as well as experiments using new starting positions (Granon and Poucet 1995), longer time delays (GisquetVerrier and Delatour 2006), or interfering events (GisquetVerrier and Delatour 2006). Over the last few years, a constellation of deficits associated with PL/IL damage has been established, such as disruption in working memory (Brito and Brito 1990; Ragozzino 2002), extinction (Morgan et al. 1993; Quirk et al. 2000), or cue-induced retrieval (Botreau et al. 2004). Although further experiments are needed to assess the validity of our new hypothesis, all these types of disruption may possibly result from a deficit in the resolution of conflict. This hypothesis opens new perspectives that offer the opportunity for understanding 
how the PL/IL area may be involved in such a wide range of cognitive processes.

\section{Materials and Methods}

\section{Animals}

Male Sprague-Dawley rats $(n=100$ from Charles River Laboratories, France), weighing $250 \mathrm{~g}$ upon arrival, were used in this experiment. They were housed in pairs in plastic cages in a temperature-controlled room $\left(21^{\circ} \mathrm{C}\right)$ on a 12 -h light/dark cycle (lights on at 07:30 a.m.). All experiments were conducted during the light phase of the cycle. The experiments were in agreement with standards of the guide for the care and use of laboratory animals (Centre National de la Recherche Scientifique-Institute for Laboratory Animal Research) and with respect to French and European Community rules (directive 86/609/EEC).

\section{Apparatus}

Experiments were conducted in an automated Y-maze made of beige PVC, comprising three arms $(13 \mathrm{~cm}$ wide $\times 60 \mathrm{~cm}$ long $\times$ $38 \mathrm{~cm}$ high) and a central hexagonal platform $(25 \mathrm{~cm})$. The floor was made up of $3 \mathrm{~mm}$-diameter steel bars, spaced at $9 \mathrm{~mm}$. Each arm was equipped with a door separating it from the central area. Food pellets ( $45 \mathrm{mg}$; Bio-Serv) were delivered by food dispensers located at each arm extremity. The arms could be illuminated by $40-\mathrm{W}$ bulbs fixed at the end of each arm.

The rats' movement was tracked by photoelectric cells located at $1 \mathrm{~cm}, 11 \mathrm{~cm}$, and $30 \mathrm{~cm}$ from the entrance of each arm. The maze was driven by a Hewlett-Packard Vectra computer, which controlled the light sequences, the door opening, and the food pellet distribution. The sequence of arms visited and latencies were recorded. The maze was located in a dimly lit room, adjacent to the colony room.

\section{Surgery}

Rats were anesthetized with $50 \mathrm{mg} / \mathrm{kg}$ ketamine hydrochloride and $20 \mathrm{mg} / \mathrm{kg}$ xylazine before being mounted in a stereotaxic frame. A midsaggital incision was made and the scalp retracted. Rats were randomly assigned to three lesioned groups (PL lesions, IL lesions, PL/IL lesions) and three sham-operated groups in Experiment 1 and to a PL-lesioned group and a sham-operated group in Experiment 2 (SHAM and PL). For lesioned rats, ibotenic acid (Sigma; concentration $63 \mathrm{mM}$, dissolved in phosphatebuffered saline) was infused bilaterally through a cannula (Plastics One; 33 gauge). The cannula was attached by a polyethylene tube (Plastics One) to a $10-\mu \mathrm{L}$ Hamilton syringe driven by a microsyringe pump (Harvard Apparatus, 11 plus). Infusions were made at a rate of $0.1 \mu \mathrm{L} / \mathrm{min}$ and the cannulae were left in place for an additional $5 \mathrm{~min}$ to allow for diffusion. The stereotaxic coordinates were adapted from the atlas of Paxinos and Watson (1986): PL lesions: $0.28 \mu \mathrm{L}$ ibotenic acid, anterior-posterior (AP): $+3.2 \mathrm{~mm}$ from bregma, mediolateral (ML): $\pm 0.75 \mathrm{~mm}$ from the midline, dorsoventral (DV): $-3.2 \mathrm{~mm}$ from dura; IL lesions: 0.13 $\mu \mathrm{L}$ ibotenic acid, AP: $+2.7 \mathrm{~mm}, \mathrm{ML}: \pm 0.75 \mathrm{~mm}$, DV: $-4.6 \mathrm{~mm}$; PL/IL lesions: $0.4 \mu \mathrm{L}$ ibotenic acid; AP: $+3.0 \mathrm{~mm}$, ML: $\pm 0.75 \mathrm{~mm}, \mathrm{DV}:-3.3 \mathrm{~mm}$. For sham-operated rats, cannulae were advanced into holes drilled above PL, IL, or PL/IL (same coordinates as above) but no solution was injected. Rats were handled regularly during the recovery period, which lasted at least $7 \mathrm{~d}$.

\section{Behavioral task}

After recovery from surgery, rats had free access to water but were food restricted to maintain their weight at $85 \%$ of their freefeeding weight.

Rats were then trained in the automated Y-maze task. This experimental design provided some unique conditions: (1) No handling was required between trials during the whole training session; (2) the goal arm of one trial became the start arm of the next trial; (3) the experimental conditions, with the exception of the reinforcement contingencies, remained strictly identical throughout the experiment. Strategy switching was thus detectable only by experiencing the task. In these experiments, four different strategies relying on two different sensory dimensions were possibly used: two response strategies (turning right or left) and two visual strategies (entering an illuminated or darkened arm). The sequence of arm illumination was pseudorandomly determined over a 200-trial sequence so that with the Light strategy, the food was distributed equally between each arm, after an equal number of left and right turns, and the lit arm never occurring more than three consecutive times with the same turn. When rats were trained with a response strategy, reinforced arms were equally distributed among the three arms and between lighted and darkened arms, with the reinforced arm never occurring more than three consecutive times in an illuminated or darkened arm.

\section{Pretraining}

During all phases of pretraining, different arms were pseudorandomly illuminated for 18 - to 22 -sec periods.

On the first day of habituation, groups of four rats were allowed to explore the maze freely for $15 \mathrm{~min}$, during which time 60 food pellets were randomly delivered to the three arms of the Y-maze. On the second day, rats were placed individually in the maze. Their entry in one arm led to the delivery of a food pellet in that arm. The second pretraining session ended when a rat was able to enter and eat the food pellet seven consecutive times. On the third and fourth day, the same procedure was used four consecutive times; then the door was closed when the rat entered an arm and it was opened after consumption of the pellet. Pretraining sessions ended when rats were able to eat their food pellets for 10 consecutive pseudotrials.

\section{Training}

Training began on the following day. During each training phase, rats were trained until they reached a criterion performance of 10 consecutive correct responses. When a rat failed to reach the criterion within 150 trials, a supplementary training session identical to the previous one was given on the following day.

\section{Experiment 1}

In the first experiment (Fig. 2), rats ( $n=75)$ were first trained with a visual strategy, before being submitted to a shift of rule, where they had to follow a response strategy, independent of the location of visual cues. Finally, rats were tested on a "reversal of strategy" where they were required to shift from a response strategy back to the visual strategy.

\section{Initial training: Light strategy}

Rats were first trained to enter the lighted arm for a food reward. When they entered an arm, the door was closed and a food pellet was delivered if the rat chose the correct arm. After 18 to $22 \mathrm{sec}$, another arm was illuminated, the door was opened, and next trial began. At the end of training, the number of right and left turns performed during the entire training session was recorded and the highest number of turns in the same direction determined their naturally preferred response (PR strategy).

\section{Rule-shifting: Light $\rightarrow$ nonpreferred response strategy}

The day after reaching criterion, rats were submitted to a ruleshifting. All aspects of the procedure remained identical to those previously described except the reinforcement contingencies. Rats were now required to always turn in the direction opposite to their preferred response (nonpreferred response: NPR), independent of the light location. 
Reversal: Nonpreferred response $\rightarrow$ preferred response strategy

On the day after, the rule was reversed and rats had to turn in the direction opposite to the one previously learned (i.e., PR) in order to obtain a food pellet.

\section{Experiment 2}

The second experiment (Fig. $3 ; n=25$ ) was designed to study the impact of the order as well as the number of strategy switchings. In this experiment, rats with PL lesions were compared to control rats and were submitted to the same first two shifts as those used in Experiment 1, but in the reverse order. They were first trained to the nonpreferred response (NPR) and then reversed to the preferred response (PR); this was followed by a rule-shift where the lit arm was reinforced. Rats were then given a second rule-shift from the lit arm to the NPR, and finally a second reversal to the PR. All other aspects of the procedure were as previously described.

\section{Initial training: nonpreferred response strategy}

Animals were initially trained to turn in the opposite direction to their preferred response corresponding to the body turn (left or right) that was used mainly during the last pretraining session.

\section{Reversal: Nonpreferred response $\rightarrow$ preferred response strategy}

On the day after, rats were submitted to a reversal and trained to turn in the opposite direction to the one used during initial training in order to get a food pellet.

\section{Rule-shifting: Preferred response $\rightarrow$ Light strategy}

Finally, on the next day rats were submitted to a rule shifting and were then required to reach the lighted arm in order to get a food pellet.

To establish whether PL lesions still impaired training after repeated strategy switching, rats from Experiment 2 were tested on two additional shifts and further trained with the NPR strategy (rule-shifting) and the PR strategy (reversal).

In the present task, each strategy is associated with different reward contingencies. When a rat is trained, for instance, to follow the light, the Light strategy is fully reinforced while the Dark strategy is unreinforced. However, as the light is half of the time on the right side and half of the time of the left side, rats using a response strategy receive $50 \%$ of reinforced trials. Consequently, and this is a common characteristic with the crossmaze tasks (Ragozzino et al. 1999a,b), for each discrimination there is always a 100\% and a 0\% reinforced strategy (the correct strategy and the reversed strategy of the same dimension), and two strategies are reinforced during half of the trials (the two strategies of the other dimension).

\section{Data analysis}

On the basis of the different arms visited by the rats, the strategies selected by the rats were identified. A strategy was considered when rats performed three or more consecutive trials following one out of the four possible strategies: Left, Right, Light, or Dark strategies. It must be noted that some overlap may occur between the response (right or left) and the visual (Light or Dark) strategies, but the overlapping can never exceed three trials. Previous experiments carried out using this apparatus only for the Light strategy (Gisquet-Verrier et al. 1989; Gisquet-Verrier and Alexinsky 1990; Botreau et al. 2004) indicated that rats began the task by a sampling phase during which they tested several of the available strategies. They then chose their naturally preferred response (PR), which gave them reinforcement on only half the trials. For this reason, rats were never trained on this strategy first.

Rats were trained until they reach the criterion of 10 consecutive correct responses. The number of trials required for CRI10 was used as an index of the rate of acquisition and also gave a correct appraisal of the task difficulty. During training rats used the correct strategy during some consecutive trials, then used another strategy, before reexperiencing the correct strategy. A way to follow their progressive acquisition is thus to consider the number of trials necessary to reach 3 (CRI3), 4 (CRI4), and finally, 10 (CRI10) consecutive correct responses. We considered that a strategy is tested when the rat uses it for at least three consecutive trials (i.e., number of trials necessary to reach CRI3). As rats learn the task by moving between strategies at the end of the period when the two strategies overlap, a chain of trials can be considered as referring to these two strategies.

For example, a rat making a series of right turns, independent of the light location, may enter the lit arm for three consecutive trials (maximal number) because the lit arm is in the right response. This may lead the rat to shift to the light strategy during the next trial, when the lit arm is on the left. Under those conditions, the three trials during which the use of the right and the Light strategy cannot be clearly evidenced will be analyzed as trials controlled by the right and the Light strategy.

We considered that a strategy is selected as the correct strategy when the rat follows this strategy during six consecutive trials (CRI6). The number of trials necessary to reach the latest criteria, and from CRI 6 to CRI 10 give a correct appraisal of the animals' capacity to maintain the correct strategy. Several parameters, illustrated in Figures 5 and 7, were recorded: (1) the first attempt for each strategy (1st), corresponded to the number of trials required before this strategy was used during three consecutive trials, (2) the maximal number of trials during which each strategy was used (CRI Max = CRI in Figs. 5 and 7), and (3) the percent of trials during which a particular strategy was selected.

All parameters were analyzed with VAR3 software (Rouanet et al. 1990). ANOVAs with the appropriate design were used. Planed comparisons were performed to test the source of significant effect or interaction.

\section{Histology}

After completion of behavioral testing, rats received a lethal dose of pentobarbital and were perfused intracardially with phosphatebuffered solution followed by a $4 \%$ formalin solution. Brains were removed, post-fixed in a formalin solution, and cryoprotected in a $30 \%$ sucrose solution. The brains were frozen, and $50-\mu \mathrm{m}$ sections were mounted on slides and stained with Cresyl Violet. The extent of the lesion was then analyzed under a microscope.

\section{Acknowledgments}

The authors thank Gérard Dutrieux for his expert technical work to build the automated task, and Pascale Veyrac and Nathalie Desvignes for the animal care. We are very grateful to Dr. Bernard Hars and Dr. Sylvie Granon for their helpful comments on an earlier version of the text. We also deeply thank Dr. Sabrina Davis for correcting the English text.

\section{References}

Beats BC, Sahakian BJ, Levy R. 1996. Cognitive performance in tests sensitive to frontal lobe dysfunction in the elderly depressed. Psychol Med 26: 591-603.

Birrell JM, Brown VJ. 2000. Medial frontal cortex mediates perceptual attentional set shifting in the rat. J Neurosci 20: 4320-4324.

Block AE, Dhanji H, Thompson-Tardif SF, Floresco SB. 2007. Thalamic-prefrontal cortical-ventral striatal circuitry mediates dissociable components of strategy set shifting. Cereb Cortex 17: $1625-1636$.

Botreau F, Gisquet-Verrier P. 2006. Memory reactivation, dissociated from behavioural expression, decreases ERK phosphorylation in the rat prefrontal cortex and amygdala. Behav Brain Res 169: 176-180.

Botreau F, El Massioui N, Cheruel F, Gisquet-Verrier P. 2004. Effects of medial prefrontal cortex and dorsal striatum lesions on retrieval processes in rats. Neuroscience 129: 539-553.

Botvinick MM, Braver TS, Barch DM, Carter CS, Cohen JD. 2001. Conflict monitoring and cognitive control. Psychol Rev 108: 624-652.

Boulougouris V, Dalley JW, Robbins TW. 2007. Effects of orbitofrontal, infralimbic and prelimbic cortical lesions on serial spatial reversal learning in the rat. Behav Brain Res 179: 219-228. 
Brito GNO, Brito LSO. 1990. Septohippocampal system and the prelimbic sector of frontal cortex: A neuropsychological battery analysis in the rat. Behav Brain Res 36: 127-146.

Brown VJ, Bowman EM. 2002. Rodent models of prefrontal cortical function. Trends Neurosci 25: 340-343.

Bussey TJ, Muir JL, Everitt BJ, Robbins TW. 1997. Triple dissociation of anterior cingulate, posterior cingulate, and medial frontal cortices on visual discrimination tasks using a touchscreen testing procedure for the rat. Behav Neurosci 111: $920-936$.

Cahill L, Haier RJ, White NS, Fallon J, Kilpatrick L, Lawrence C, Potkin SG, Alkire MT. 2001. Sex-related difference in amygdala activity during emotionally influenced memory storage. Neurobiol Learn Mem 75: 1-9.

Carter CS, van Veen V. 2007. Anterior cingulate cortex and conflict detection: An update of theory and data. Cogn Affect Behav Neurosci 7: 367-379.

Chudasama Y, Robbins TW. 2003. Dissociable contributions of the orbitofrontal and infralimbic cortex to pavlovian autoshaping and discrimination reversal learning: Further evidence for the functional heterogeneity of the rodent frontal cortex. J Neurosci $\mathbf{2 3}$ 8771-8780.

Dalley JW, Cardinal RN, Robbins TW. 2004. Prefrontal executive and cognitive functions in rodents: Neural and neurochemical substrates. Neurosci Biobehav Rev 28: 771-784.

De Bruin JP, Feenstra MG, Broersen LM, Van Leeuwen M, Arens C, De Vries S, Joosten RN. 2000. Role of the prefrontal cortex of the rat in learning and decision making: Effects of transient inactivation. Prog Brain Res 126: 103-113.

Delatour B. 2000. Quel apport de la neuroanatomie fonctionnelle à l'étude des homologies entre les différentes espèces? L'exemple du cortex préfrontal. Ann Fondat Fyssen 14: 76-85.

Delatour B, Gisquet-Verrier P. 1996. Prelimbic cortex specific lesions disrupt delayed-variable response tasks in the rat. Behav Neurosci 110: $1282-1298$

Delatour B, Gisquet-Verrier P. 1999. Lesions of the prelimbic-infralimbic cortices in rats do not disrupt response selection processes but induce delay-dependent deficits: Evidence for a role in working memory? Behav Neurosci 113: 941-955

Delatour B, Gisquet-Verrier P. 2000. Functional role of rat prelimbicinfralimbic cortices in spatial memory: Evidence for their involvement in attention and behavioural flexibility. Behav Brain Res 109: $113-128$.

Deweer B, Ergis AM, Fossati P, Pillon B, Boller F, Agid Y, Dubois B. 1994 Explicit memory, procedural learning and lexical priming in Alzheimer's disease. Cortex 30: 113-126.

Dias R, Aggleton JP. 2000. Effects of selective excitotoxic prefrontal lesions on acquisition of nonmatching- and matching-to-place in the T-maze in the rat: Differential involvement of the prelimbicinfralimbic and anterior cingulate cortices in providing behavioural flexibility. Eur J Neurosci 12: 4457-4466.

Dias R, Robbins TW, Roberts AC. 1996. Primate analogue of the Wisconsin Card Sorting Test: Effects of excitotoxic lesions of the prefrontal cortex in the marmoset. Behav Neurosci 110: $872-886$.

Egner T, Hirsch J. 2005. Cognitive control mechanisms resolve conflict through cortical amplification of task-relevant information. Nat Neurosci 8: $1784-1790$.

Ferry AT, Lu XC, Price JL. 2000. Effects of excitotoxic lesions in the ventral striatopallidal-thalamocortical pathway on odor reversal learning: Inability to extinguish an incorrect response. Exp Brain Res 131: $320-335$.

Floresco SB, Ghods-Sharifi S. 2007. Amygdala-prefrontal cortical circuitry regulates effort-based decision making. Cereb Cortex 17: 251-260.

Floresco SB, Block AE, Tse MT. 2008. Inactivation of the medial prefrontal cortex of the rat impairs strategy set-shifting, but not reversal learning, using a novel, automated procedure. Behav Brain Res 190: 85-96.

Floresco SB, Zhang Y, Enomoto T. 2009. Neural circuits subserving behavioral flexibility and their relevance to schizophrenia. Behav Brain Res 204: 396-409.

Fossati P, Amar G, Raoux N, Ergis AM, Allilaire JF. 1999. Executive functioning and verbal memory in young patients with unipolar depression and schizophrenia. Psychiatry Res 89: 171-187.

Gisquet-Verrier P, Alexinsky T. 1990. Long-term spontaneous improvement of performance is related to the strength of the initial training: Theoretical implications. Behav Neural Biol 53: 298-304.

Gisquet-Verrier P, Delatour B. 2006. The role of the rat prelimbic/ infralimbic cortex in working memory: Not involved in the short-term maintenance but in monitoring and processing functions. Neuroscience 141: 585-596.

Gisquet-Verrier P, Dekeyne A, Alexinsky T. 1989. Differential effects of several retrieval cues over time: Evidence for time-dependent reorganization of memory. Anim Learn Behav 17: 394-408.
Gisquet-Verrier P, Winocur G, Delatour B. 2000. Functional dissociation between dorsal and ventral regions of the medial prefrontal cortex in rats. Psychobiology 28: 248-260.

Gisquet-Verrier P, Botreau F, Venero C, Sandi C. 2004. Exposure to retrieval cues improves retention performance and induces changes in ACTH and corticosterone release. Psychoneuroendocrinology 29: $529-556$.

Goldberg TE, Weinberger DR. 1988. Probing prefrontal function in schizophrenia with neuropsychological paradigms. Schizophr Bull 14: 179-183.

Granon S, Poucet B. 1995. Medial prefrontal lesions in the rat and spatial navigation: Evidence for impaired planning. Behav Neurosci 109: 474-484.

Granon S, Vidal C, Thinus-Blanc C, Changeux JP, Poucet B. 1994. Working memory, response selection, and effortful processing in rats with medial prefrontal lesions. Behav Neurosci 108: 883-891.

Haddon JE, Killcross S. 2006. Prefrontal cortex lesions disrupt the contextual control of response conflict. J Neurosci 26: 2933-2940.

Hoover WB, Vertes RP. 2007. Anatomical analysis of afferent projections to the medial prefrontal cortex in the rat. Brain Struct Funct 212: $149-179$.

Joel D, Weiner I, Feldon J. 1997. Electrolytic lesions of the medial prefrontal cortex in rats disrupt performance on an analog of the Wisconsin Card Sorting Test, but do not disrupt latent inhibition: Implications for animal models of schizophrenia. Behav Brain Res 85: 187-201.

Kalivas PW, O'Brien C. 2008. Drug addiction as a pathology of staged neuroplasticity. Neuropsychopharmacology 33: 166-180.

Killcross S, Coutureau E. 2003. Coordination of actions and habits in the medial prefrontal cortex of rats. Cereb Cortex 13: 400-408.

Kim J, Ragozzino ME. 2005. The involvement of the orbitofrontal cortex in learning under changing task contingencies. Neurobiol Learn Mem 83: $125-133$

Kinoshita S, Yokoyama C, Masaki D, Yamashita T, Tsuchida H, Nakatomi Y, Fukui K. 2008. Effects of rat medial prefrontal cortex lesions on olfactory serial reversal and delayed alternation tasks. Neurosci Res 60: 213-218

Kolb B, Nonneman AJ, Singh RK. 1974. Double dissociation of spatial impairments and perseveration following selective prefrontal lesions in rats. J Comp Physiol Psychol 87: 772-780.

Krettek JE, Price JL. 1977. The cortical projections of the mediodorsal nucleus and adjacent thalamic nuclei in the rat. J Comp Neurol 171: 157-192.

Lawrence V, Houghton S, Douglas G, Durkin K, Whiting K, Tannock R. 2004. Executive function and ADHD: A comparison of children's performance during neuropsychological testing and real-world activities. J Atten Disord 7: 137-149.

Lebron K, Milad MR, Quirk GJ. 2004. Delayed recall of fear extinction in rats with lesions of ventral medial prefrontal cortex. Learn Mem 11: 544-548.

Li L, Shao J. 1998. Restricted lesions to ventral prefrontal subareas block reversal learning but not visual discrimination learning in rats. Physiol Behav 65: 371-379.

Mansouri FA, Tanaka K, Buckley MJ. 2009. Conflict-induced behavioural adjustment: A clue to the executive functions of the prefrontal cortex. Nat Rev Neurosci 10: 141-152.

Marquis JP, Killcross S, Haddon JE. 2007. Inactivation of the prelimbic, but not infralimbic, prefrontal cortex impairs the contextual control of response conflict in rats. Eur J Neurosci 25: 559-566.

McAlonan K, Brown VJ. 2003. Orbital prefrontal cortex mediates reversal learning and not attentional set shifting in the rat. Behav Brain Res 146: 97-103.

McDonald RJ, Foong N, Ray C, Rizos Z, Hong NS. 2007. The role of medial prefrontal cortex in context-specific inhibition during reversal learning of a visual discrimination. Exp Brain Res 177: 509-519.

Merriam EP, Thase ME, Haas GL, Keshavan MS, Sweeney JA. 1999. Prefrontal cortical dysfunction in depression determined by Wisconsin Card Sorting Test performance. Am J Psychiatry 156: 780-782.

Milad MR, Quirk GJ. 2002. Neurons in medial prefrontal cortex signal memory for fear extinction. Nature 420: 70-74.

Milner B. 1963. Effect of different brain lesions on card sorting. Arch Neurol 9: $90-100$.

Morgan MA, Romanski LM, LeDoux JE. 1993. Extinction of emotional learning: Contribution of medial prefrontal cortex. Neurosci Lett 163: $109-113$

Morice R. 1990. Cognitive inflexibility and pre-frontal dysfunction in schizophrenia and mania. Br J Psychiatry 157: 50-54.

Murphy ER, Dalley JW, Robbins TW. 2005. Local glutamate receptor antagonism in the rat prefrontal cortex disrupts response inhibition in a visuospatial attentional task. Psychopharmacology (Berl) 179: 99-107. 
Owen AM, Roberts AC, Polkey CE, Sahakian BJ, Robbins TW. 1991. Extradimensional versus intra-dimensional set shifting performance following frontal lobe excisions, temporal lobe excisions or amygdalohippocampectomy in man. Neuropsychologia 29: 993-1006.

Paxinos G, Watson C. 1986. The rat brain in stereotaxic coordinates. Academic Press, San Diego, CA.

Peters J, Vallone J, Laurendi K, Kalivas PW. 2008. Opposing roles for the ventral prefrontal cortex and the basolateral amygdala on the spontaneous recovery of cocaine-seeking in rats. Psychopharmacology (Berl) 197: 319-326.

Preuss TM. 1995. Do rats have prefrontal cortex? The Rose-Woolsey-Akert program reconsidered. J Cogn Neurosci 7: 1-24.

Quirk GJ, Beer JS. 2006. Prefrontal involvement in the regulation of emotion: Convergence of rat and human studies. Curr Opin Neurobiol 16: 723-727.

Quirk GJ, Russo GK, Barron JL, Lebron K. 2000. The role of ventromedial prefrontal cortex in the recovery of extinguished fear. J Neurosci 20: 6225-6231.

Ragozzino ME. 2002. The effects of prelimbic and infralimbic lesions on working memory for visual objects in rats. Neurobiol Learn Mem 77: $29-43$.

Ragozzino M. 2007. The contribution of the medial prefrontal cortex, orbitofrontal cortex and dorsomedial striatum to behavioral flexibility. Ann N Y Acad Sci 1121: 355-375.

Ragozzino ME, Detrick S, Kesner RP. 1999a. Involvement of the prelimbic-infralimbic areas of the rodent prefrontal cortex in behavioral flexibility for place and response learning. J Neurosci 19: 4585-4594.

Ragozzino ME, Wilcox C, Raso M, Kesner RP. 1999b. Involvement of rodent prefrontal cortex subregions in strategy switching. Behav Neurosci 113: 32-41.

Ragozzino ME, Kim J, Hassert D, Minniti N, Kiang C. 2003. The contribution of the rat prelimbic-infralimbic areas to different forms of task switching. Behav Neurosci 117: 1054-1065.

Rich EL, Shapiro ML. 2007. Prelimbic/infralimbic inactivation impairs memory for multiple task switches, but not flexible selection of familiar tasks. J Neurosci 27: 4747-4755.

Rich EL, Shapiro M. 2009. Rat prefrontal cortical neurons selectively code strategy switches. J Neurosci 29: 7208-7219.

Roberts AC, Robbins TW, Everitt BJ. 1988. The effects of intradimensional and extradimensional shifts on visual discrimination learning in humans and non-human primates. Q J Exp Psychol B 40: 321-341.

Rouanet H, Bernard JM, Leroux B. 1990. Statistiques en sciences humaines: Analyse inductive des données. Dunot, Paris.

Salazar RF, White W, Lacroix L, Feldon J, White IM. 2004. NMDA lesions in the medial prefrontal cortex impair the ability to inhibit responses during reversal of a simple spatial discrimination. Behav Brain Res 152: 413-424.

Seamans JK, Floresco SB, Phillips AG. 1995. Functional differences between the prelimbic and anterior cingulate regions of the rat prefrontal cortex. Behav Neurosci 109: 1063-1073.

Sierra-Mercado D Jr, Corcoran KA, Lebron-Milad K, Quirk GJ. 2006. Inactivation of the ventromedial prefrontal cortex reduces expression of conditioned fear and impairs subsequent recall of extinction. Eur J Neurosci 24: 1751-1758.

Stefani MR, Groth K, Moghaddam B. 2003. Glutamate receptors in the rat medial prefrontal cortex regulate set-shifting ability. Behav Neurosci 117: $728-737$.

Tait DS, Marston HM, Shahid M, Brown VJ. 2009. Asenapine restores cognitive flexibility in rats with medial prefrontal cortex lesions. Psychopharmacology (Berl) 202: 295-306.

Van Eden CG, Uylings HB. 1985. Cytoarchitectonic development of the prefrontal cortex in the rat. J Comp Neurol 241: 253-267.

van Veen V, Cohen JD, Botvinick MM, Stenger VA, Carter CS. 2001. Anterior cingulate cortex, conflict monitoring, and levels of processing. Neuroimage 14: 1302-1308.

Vidal-Gonzalez I, Vidal-Gonzalez B, Rauch SL, Quirk GJ. 2006. Microstimulation reveals opposing influences of prelimbic and infralimbic cortex on the expression of conditioned fear. Learn Mem 13: $728-733$.

Wise SP, Murray EA, Gerfen CR. 1996. The frontal cortex-basal ganglia system in primates. Crit Rev Neurobiol 10: 317-356.

Wittfoth M, Schardt DM, Fahle M, Herrmann M. 2009. How the brain resolves high conflict situations: Double conflict involvement of dorsolateral prefrontal cortex. Neuroimage 44: 1201-1209.

Young JJ, Shapiro ML. 2009. Double dissociation and hierarchical organization of strategy switches and reversals in the rat PFC. Behav Neurosci 123: 1028-1035.

Received May 4, 2010; accepted in revised form September 28, 2010. 


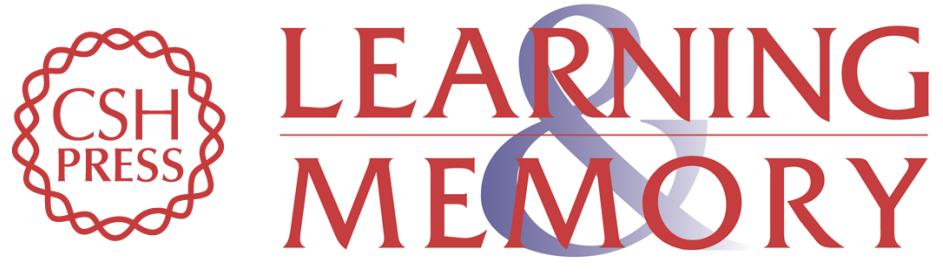

\section{The differential involvement of the prelimbic and infralimbic cortices in response conflict affects behavioral flexibility in rats trained in a new automated strategy-switching task}

Catherine Oualian and Pascale Gisquet-Verrier

Learn. Mem. 2010, 17:

Access the most recent version at doi: $10.1101 / \mathrm{lm} .1858010$

References This article cites 82 articles, 10 of which can be accessed free at: http://learnmem.cshlp.org/content/17/12/654.full.html\#ref-list-1

License

Email Alerting

Receive free email alerts when new articles cite this article - sign up in the box at the Service top right corner of the article or click here. 